\title{
Supporting Information of
}

\section{Uniform Biodegradable Fiber-Like Micelles and Block Co-micelles via 'Living' Crystallization-Driven Self-Assembly of Poly $(L$-lactide) Block Copolymers: The Importance of Reducing Unimer Self-Nucleation via Hydrogen Bond Disruption}

Yunxiang He, ${ }^{1}$ Jean-Charles Eloi, ${ }^{2}$ Robert L. Harniman, ${ }^{2}$ Robert M. Richardson, ${ }^{3}$ George R. Whittell, ${ }^{1}$ Robert T. Mathers, ${ }^{4}$ Andrew P. Dove,${ }^{{ }^{*}}$ Rachel K. O’Reilly, ${ }^{5^{*}}$ and Ian Manners ${ }^{1,6^{*}}$

${ }^{1}$ School of Chemistry, University of Bristol, Bristol BS8 1TS, UK

${ }^{2}$ Chemical Imaging Facility, School of Chemistry, University of Bristol, Bristol BS8 1TS, UK ${ }^{3}$ School of Physics, University of Bristol, Tyndall Avenue, Bristol, BS8 1TL, UK

${ }^{4}$ Department of Chemistry, Pennsylvania State University, New Kensington, PA 15068, USA ${ }^{5}$ School of Chemistry, University of Birmingham, Edgbaston, Birmingham, B15 2TT, UK ${ }^{6}$ Department of Chemistry, University of Victoria, Victoria, BC V8W 3V6, Canada *To whom correspondence should be addressed:

a.dove@bham.ac.uk

r.oreilly@bham.ac.uk

imanners@uvic.ca 


\section{Materials and Methods}

All reactions were carried out in an MBraun MB150B-G glove box under nitrogen atmosphere or using standard Schlenk line techniques. Solvents for self-assembly were purchased at HPLC grade and filtered through a PTFE membrane with pore size of $450 \mathrm{~nm}$. Solvents for reactions were obtained from a Grubbs type solvent purification system. All reagents and solvents were purchased from Sigma-Aldrich (UK), Acros, Fluka, Fisher Chemical and Alfa Aesar, and used as received unless otherwise noted. $L$-Lactide were purified by azeotropic distillation or recrystallization respectively from toluene, followed by drying at $50{ }^{\circ} \mathrm{C}$ under reduced pressure over night prior to use. $N$-Isopropylacrylamide (NIPAm) was recrystallized twice from methanol before use. 2,2'-Azobis(2-methylpropionitrile) (AIBN) was recrystallized twice from methanol and stored in the dark at $4{ }^{\circ} \mathrm{C}$. 2-Vinylpyridine (2VP) was passed through a basic aluminum oxide column before use. 1,8-Diazabicyclo[5.4.0]undec-7-ene (DBU) were dried over $\mathrm{CaH}_{2}$ and distilled under vacuum before use.

Matrix-assisted laser desorption/ionization time of flight (MALDI-TOF) mass spectrometry measurements were performed using a Bruker Ultraflextreme running in linear mode. Samples were prepared using a trans-2-[3-(4-tert-butylphenyl)-2-methyl-2propenylidene]malononitrile matrix $(20 \mathrm{mg} / \mathrm{mL}$ in THF) and the polymer sample $(2 \mathrm{mg} / \mathrm{mL}$ in THF), mixed in a 10:1 $(v / v)$ ratio. Approximately $1 \mu \mathrm{L}$ of the mixed solution was deposited onto a stainless steel sample plate and allowed to dry in air.

${ }^{1} \mathrm{H}$ and ${ }^{13} \mathrm{C}$ NMR spectra were obtained using a Varian $400 \mathrm{MHz}$ spectrometer with $\mathrm{CDCl}_{3}\left({ }^{1} \mathrm{H}\right.$ NMR: $\delta=7.26 \mathrm{ppm} ;{ }^{13} \mathrm{C}$ NMR $\delta=77.16 \mathrm{ppm}$ ) as solvents. DOSY NMR spectra were obtained using a Varian $500 \mathrm{MHz}$ spectrometer with $\mathrm{CDCl}_{3}$ as solvents.

Infrared spectra (IR) were recorded on a Perkin Elmer Spectrum One Fourier Transform Infrared Spectrometer (FT-IR).

Thermogravimetric analysis (TGA) was performed on a TA Instruments Q100 calorimeter at a scan rate of $10^{\circ} \mathrm{C} / \mathrm{min}$ under nitrogen.

Gel Permeation Chromatography (GPC) was conducted on a Viscotek VE2001 GPCmax chromatograph equipped with a refractive indices (RI) and a UV detector array. $n$-Bu $4 \mathrm{NBr} / \mathrm{THF}$ $(0.1 \mathrm{w} / \mathrm{w} \%)$ was used as the eluent, with the flow rate set at $1 \mathrm{~mL} / \mathrm{min}$. The columns used were of grade GP5000HHR followed by GP2500HHR (Viscotek) at a constant temperature of $30{ }^{\circ} \mathrm{C}$. The calibration of RI detector was carried out using polystyrene standards (Viscotek). Samples 
were prepared at $2 \mathrm{mg} / \mathrm{mL}$ in eluent and filtered through a Ministart SRP 15 filter (polytetrafluorethylene membrane, pore size $=0.45 \mu \mathrm{m}$ ).

Dynamic light scattering (DLS) was employed to determine the size of micelles formed in selective solvents. The measurements were performed at $25{ }^{\circ} \mathrm{C}$ on a Malvern Instruments Zetasizer Nano S using a $5 \mathrm{~mW} \mathrm{He}-\mathrm{Ne}$ laser $(633 \mathrm{~nm})$ and a detector oriented at $173^{\circ}$. Samples $(1 \mathrm{~mL})$ were measured in an optical glass cuvette $(10.0 \mathrm{~mm}$ path length) with a concentration of $0.05 \mathrm{mg} / \mathrm{mL}$. The results of DLS studies are reported as apparent hydrodynamic radii ( $\left.\mathrm{R}_{\mathrm{h} \text {,app }}\right)$, acknowledging that the particles have been modelled as spheres in the experiments conducted.

Transmission electron microscopy (TEM) images were obtained on a JSM-1400 micrpscope (JEOL Japan) with a Orius Sc200 camera from Gatan, USA, which was operated at $120 \mathrm{kV}$. Selected area electron diffraction (SAED) data and scanning transmission election microscopy (STEM) images were obtained on a JSM-2100F microscope (JEOL Japan) with a Orius SC1000 camera from Gatan USA. STEM was operated in high angle annular dark field imaging (HAADF) mode. Samples were prepared by drop casting $4 \mu \mathrm{L}$ of the micelle solution onto a carbon coated copper grid. Copper grids (400 mesh) were purchased from Agar Scientific and carbon films were prepared on mica sheets by carbon sputtering with an Agar TEM Turbo Carbon Coater. The carbon films were deposited onto the copper grids by floatation on water and the carbon coated grids were allowed to dry in air. For TEM images, samples were stained with uranyl acetate solution (2\% wt in EtOH). For STEM and SAED data, no staining was applied.

For micelle contour lengths analysis, ca. 200 micelles in several images were traced manually using the ImageJ software package developed at the US National Institute of Health. The number average micelle length $\left(L_{\mathrm{n}}\right)$ and weight average micelle length $\left(L_{\mathrm{w}}\right)$ were calculated using eq. $\mathrm{S} 1$ from measurements of the contour lengths $\left(L_{\mathrm{i}}\right)$ of individual micelles, where $N_{\mathrm{i}}$ is the number of micelles of length $L_{\mathrm{i}}$, and $n$ is the number of micelles examined in each sample. The distribution of micelle lengths is characterized by both $L_{\mathrm{w}} / L_{\mathrm{n}}$ and the ratio of standard deviation $/ L_{\mathrm{n}}\left(\sigma / L_{\mathrm{n}}\right)$.

$L_{n}=\frac{\sum_{i=1}^{n} N_{i} L_{i}}{\sum_{i=1}^{n} N i} \quad L_{w}=\frac{\sum_{i=1}^{n} N_{i} L_{i}^{2}}{\sum_{i=1}^{n} N i L i} \quad$ (eq.S1)

Atomic force microscopy (AFM) analyses were performed in ambient conditions using a Bruker Multimode VIII atomic force microscope equipped with a ScanAsyst-HR fast scanning module and a ScanAsyst-Air-HR probe (tip radius, $2 \mathrm{~nm}$ ), utilising peak force feedback control. 
Samples for AFM were prepared by drop casting $6 \mu \mathrm{L}$ of micelle colloidal solution onto freshly cleaved mica before drying with a gentle stream of nitrogen.

X-ray scattering measurements (small- and wide-angle, SAXS and WAXS, respectively) were performed in transmission geometry using a Ganesha small angle X-ray scattering apparatus (SAXSLAB, Denmark). Solution samples were sealed into $1.5 \mathrm{~mm}$ diameter quartz capillary tubes (Capillary Tube Supplies, Cornwall, UK) and solid-state samples were drop cast onto mica or Kapton film (4,4'-oxydiphenylene-pyromellitimide, DuPont). The capillary or film was then secured in position, perpendicular to the X-ray beam and the detector was positioned at a distance of $1050 \mathrm{~mm}$ and $100 \mathrm{~mm}$ for the SAXS and WAXS measurements respectively. All measurements were recorded after evacuating the chamber to reduce air scattering. All the SAXS data was analyzed after applying corrections for the scattering from the solvents and the empty capillary tube. SAXGUI (from SAXSLAB) was used for empty cell correction and SIMPLE SUBTRACT (in-house) for solvent.

Computation of octanol-water partition coefficient normalized by the Connolly surface area $\left(\log \mathrm{P}_{\text {oct }} / \mathrm{SA}\right)$ values. $\log \mathrm{P}_{\text {oct }} / \mathrm{SA}$ values were calculated with a similar reported method. ${ }^{1}$ $\log \mathrm{P}_{\text {oct }} / \mathrm{SA}$ values were calculated with the ALogP98 method in Materials Studio 2019. The surface area was calculated after minimizing the energy of the solvents with the Forcite Molecular Dynamics module in Materials Studio 2019.

\section{Synthesis procedures}

Synthesis of 4-cyano-4-(((phenethylthio)carbonothioyl)thio)pentanoic acid (CTAСООН). Following previously reported procedures, ${ }^{2-3}$ in an oven-dried round bottom flask, $2-$ Phenylethanethiol ( $4.0 \mathrm{~mL}, 29.86 \mathrm{mmol})$ was added dropwise to a stirred suspension of $\mathrm{K}_{3} \mathrm{PO}_{4}$ $(8.0 \mathrm{~g}, 37.69 \mathrm{mmol})$ in acetone $(20 \mathrm{~mL})$ and stirring for $1 \mathrm{~h} . \mathrm{CS}_{2}(5.5 \mathrm{~mL}, 91.45 \mathrm{mmol})$ was added and the solution turned bright yellow. After stirring for $16 \mathrm{~h}$, the suspension was filtered, and the cake was washed with acetone $(2 \times 20 \mathrm{~mL})$. After removing the solvents from the filtrate under reduced pressure, the resulting yellow solid was suspended in diethyl ether (100 $\mathrm{mL})$. Solid iodine $(3.2 \mathrm{~g}, 12.61 \mathrm{mmol})$ was gradually added and then stirred at room temperature for $1 \mathrm{~h}$, and the insoluble white precipitate was removed by filtration. The yellowbrown filtrate was washed with an aqueous solution of sodium thiosulfate, dried over magnesium sulfate, and then evaporated to yield yellow solid. 4,4'-azobis(4-cyanopentanoic acid) (ACVA) (5.1 g, $17.90 \mathrm{mmol}$ ) was added to a solution of the solid in ethyl acetate (50 $\mathrm{mL}$ ). The solution was degassed by nitrogen bubbling for $30 \mathrm{~min}$ and heated at reflux under 
nitrogen for $16 \mathrm{~h}$. After removal of the solvents under reduced pressure, the crude product was washed with water $(5 \times 100 \mathrm{~mL})$. The organic phase was concentrated and purified by silica chromatography using a mixed eluent (hexane: ethyl acetate $=4: 1$, gradually increasing to $1: 1$ ) to afford CTA-COOH as an orange oil (4.3 g, 44\%). ${ }^{1} \mathrm{H}$ NMR (400 $\left.\mathrm{MHz}, \mathrm{CDCl}_{3}, 298 \mathrm{~K}\right)$ : $\delta$ (ppm) 7.4-7.2 (m, 5H, Ph), $3.60\left(\mathrm{t}, 2 \mathrm{H}, \mathrm{PhCH}_{2} \underline{\mathrm{CH}}_{2}\right), 3.00\left(\mathrm{t}, 2 \mathrm{H}, \mathrm{PhCH}_{2}\right), 2.70$ (t, $2 \mathrm{H}$, $\left.\mathrm{C}_{2} \mathrm{COOH}\right), 2.6-2.4\left(\mathrm{~m}, 2 \mathrm{H}, \mathrm{CNCC}_{2}\right), 1.90\left(\mathrm{~s}, 3 \mathrm{H}, \mathrm{CH}_{3}\right) .{ }^{13} \mathrm{C} \mathrm{NMR}\left(125 \mathrm{MHz}, \mathrm{CDCl}_{3}, 298\right.$ $\mathrm{K}): \delta(\mathrm{ppm}) 216.5(\underline{\mathrm{C}}=\mathrm{S}), 177.2(\underline{\mathrm{C}}=\mathrm{O}), 139.2,128.9,128.6$, and $126.9(\mathrm{Ph}), 118.9(\mathrm{CN}), 46.4$ $(\underline{\mathrm{CCN}}), 38.1\left(\underline{\mathrm{CH}}_{2} \mathrm{CS}\right), 34.2\left(\mathrm{PhCH}_{2}\right), 33.6\left(\mathrm{CNCCH}_{2}\right), 29.6\left(\underline{\mathrm{CH}}_{2} \mathrm{COOH}\right), 24.9\left(\underline{\mathrm{CH}}_{3}\right)$.

\section{Synthesis of 6-hydroxyhexyl 4-cyano-4(((phenethylthio)carbonothioyl)thio)pentanoate}

(CTA-OH). In an oven-dried Schlenk flask, CTA-COOH (1.0 g, $3.24 \mathrm{mmol})$ and 1,6hexanediol (3.0 g, $25.92 \mathrm{mmol})$ were dissolved in dry $\mathrm{CHCl}_{3}(50 \mathrm{~mL}) . \quad N-(3-$ dimethylaminopropyl)- $N$ '-ethylcarbodiimide hydrochloride (EDC $\cdot \mathrm{HCl})(0.95 \mathrm{~g}, 4.87 \mathrm{mmol})$ and DMAP (59 mg, $0.487 \mathrm{mmol})$ were dissolved in dry $\mathrm{CHCl}_{3}(15 \mathrm{~mL})$ in another oven-dried Schlenk flask at ambient temperature, followed by adding into the reaction flask via a syringe. The reaction mixture was stirred under reflux for $48 \mathrm{~h}$, filtered and concentrated to yield orange oil residue. The crude product was purified by silica chromatography (hexane: ethyl acetate = 3:1 as eluent) to afford CTA-OH as an orange oil (870 mg, $61 \%) .{ }^{1} \mathrm{H}$ NMR $\left(400 \mathrm{MHz}, \mathrm{CDCl}_{3}\right.$, $298 \mathrm{~K}): \delta(\mathrm{ppm})$ 7.4-7.2 (m, 5H, Ph), $4.12\left(\mathrm{t}, 2 \mathrm{H}, \mathrm{COOC}_{2}\right), 3.66\left(\mathrm{t}, 2 \mathrm{H}, \mathrm{C}_{2} \mathrm{OH}\right), 3.59(\mathrm{t}, 2 \mathrm{H}$, $\mathrm{PhC}_{2} \mathrm{CH}_{2}$ ), 3.00 (t, 2H, Ph- $\left.\underline{\mathrm{H}}_{2}\right), 2.64$ (t, 2H, $\left.\underline{\mathrm{CH}}_{2}-\mathrm{COOH}\right), 2.6-2.4$ (m, 2H, CN-C-C $\left.\underline{\mathrm{H}}_{2}\right), 1.90$ (s, 3H, $\left.\underline{\mathrm{C}}_{3}\right), 1.7-1.4$ (m, 8H, $\left.\underline{\mathrm{C}}_{2} \mathrm{C}_{2} \underline{\mathrm{C}}_{2} \underline{\mathrm{C}}_{2} \underline{\mathrm{CH}}_{2} \mathrm{OH}\right) .{ }^{13} \mathrm{C} \mathrm{NMR}\left(125 \mathrm{MHz}, \mathrm{CDCl}_{3}, 298 \mathrm{~K}\right)$ : $\delta(\mathrm{ppm}) 216.5(\mathrm{C}=\mathrm{S}), 171.6(\mathrm{C}=\mathrm{O}), 139.2,128.9,128.6$, and $126.9(\mathrm{Ph}), 118.9(\mathrm{CN}), 65.3$ $\left(\mathrm{COO} \underline{C H}_{2}\right), 62.9\left(\underline{\mathrm{CH}}_{2} \mathrm{OH}\right), 46.4(\underline{\mathrm{CCN}}), 38.1\left(\underline{\mathrm{CH}}_{2} \mathrm{CS}\right), 34.2\left(\mathrm{PhCH}_{2}\right), 34.1\left(\mathrm{CNCCH}_{2}\right), 32.7$ $\left(\underline{\mathrm{CH}}_{2} \mathrm{CH}_{2} \mathrm{OH}\right), 30.6\left(\underline{\mathrm{CH}_{2} \mathrm{COOH}}\right), 28.7 \quad\left(\mathrm{COOCH}_{2} \underline{\mathrm{CH}}_{2}\right), 25.8 \quad\left(\mathrm{COOCH}_{2} \mathrm{CH}_{2} \underline{\mathrm{CH}}_{2}\right), 25.5$ $\left(\mathrm{CH}_{2} \mathrm{CH}_{2} \mathrm{CH}_{2} \mathrm{OH}\right), 24.9\left(\underline{\mathrm{CH}}_{3}\right)$.

Synthesis of CTA-PLLA47. In a nitrogen-filled glove box, solutions of DBU (11.2 uL, 0.18 $\mathrm{mmol}$ ) and CTA-OH (28 mg, $0.062 \mathrm{mmol})$ in dry DCM (2 mL) were added to solution of $L$ lactide (400 mg, $2.78 \mathrm{mmol})$ in dry DCM $(1 \mathrm{~mL})$. After stirring for $1 \mathrm{~min}$ at room temperature, the solution was quenched with benzoic acid and stirred for $30 \mathrm{~min}$. After removed from the glove box, the reaction solution was precipitated three times into $\mathrm{MeOH}$ and collected by centrifugation. The Polymer was further dried in a vacuum oven for $16 \mathrm{~h}$ before characterization (367 mg, 92\%). ${ }^{1} \mathrm{H}$ NMR (400 MHz, $\mathrm{CDCl}_{3}$ ): $\delta$ (ppm) 5.17 (q, 102H, $\mathrm{CHCH}_{3}$ ), 1.57 (d, 306H, $\left.\mathrm{CHCH}_{3}\right) \cdot \mathrm{M}_{\mathrm{n}}(\mathrm{NMR}): 7698 \mathrm{~g} \cdot \mathrm{mol}^{-1} . \mathrm{MALDI}: \mathrm{m} / \mathrm{z}=7232, \mathrm{DP}_{\mathrm{n}}=47 . \mathrm{GPC}(n-$ 
$\mathrm{Bu}_{4} \mathrm{NBr} / \mathrm{THF}$, PS standard): $M_{\mathrm{n}}=10,200 \mathrm{~g} \cdot \mathrm{mol}^{-1}, \bigoplus_{\mathrm{m}}=1.09 . v_{\max }($ neat $) / \mathrm{cm}^{-1}: 3000-2880(\mathrm{C}-$ $\mathrm{H}) ; 1755,1044(\mathrm{C}=\mathrm{O}) ; 1456\left(\mathrm{CH}_{3}\right) ; 1210-1163(\mathrm{C}(\mathrm{O})-\mathrm{O}) ; 1088(\mathrm{C}-\mathrm{O})$.

Synthesis of PLLA47- $\boldsymbol{b}$-PNIPAm267. CTA-PLLA47 (100 mg, 0.015 mmol), NIPAm (850 mg, $7.5 \mathrm{mmol}$ ) and AIBN (0.47 mg, $0.003 \mathrm{mmol})$ were dissolved in 1,4-dioxane ( $3 \mathrm{~mL}$ ) in a Schlenk flask. The solution was then freeze-pump-thawed four times and heated for $3.5 \mathrm{~h}$ at $70{ }^{\circ} \mathrm{C}$. The reaction was quenched by immersion of the ampoule in liquid nitrogen and the block copolymer was precipitated in hexane three times, followed by drying under vacuum $(403 \mathrm{mg}$, 42\%). DP of PNIPAm was confirmed by ${ }^{1} \mathrm{H}$ NMR spectrum based on CTA-PLLA DP according to MALDI-TOF spectrometry. ${ }^{1} \mathrm{H}$ NMR (400 MHz, $\mathrm{CDCl}_{3}$ ): $\delta$ (ppm) 7.0-5.6 (br,

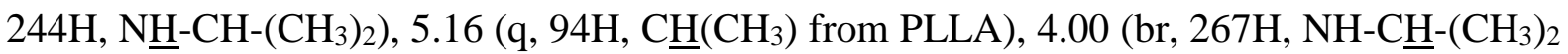
from PNIPAm), 2.3-0.7 (3673 H, m, $\underline{\mathrm{C}}_{3}$ from PNIPAm, $\mathrm{CH}\left(\mathrm{C}_{3}\right)$ from PLLA, $\underline{\mathrm{CHCH}}_{2}$ from PNIPAm). GPC $\left(n-\mathrm{Bu}_{4} \mathrm{NBr} / \mathrm{THF}\right.$, PS standard $): M_{\mathrm{n}}=180,600 \mathrm{~g} \cdot \mathrm{mol}^{-1}, \emptyset_{\mathrm{m}}=1.11 . v_{\max }$ (neat)/cm ${ }^{-1}: 3510-3313(\mathrm{~N}-\mathrm{H}) ; 3000-2880(\mathrm{C}-\mathrm{H}) ; 1755(\mathrm{C}=\mathrm{O}) ; 1644(\mathrm{NH}-\mathrm{C}=\mathrm{O}) ; 1458\left(\mathrm{CH}_{3}\right)$; 1386-1367 (C-H), 1276-1185 (C-O); 1130,1088 (C-O).

Synthesis of PLLA47- $\boldsymbol{b}$-P2VP503. PLLA 47 (100 mg, $0.015 \mathrm{mmol}), 2$ VP (886 $\mu \mathrm{L}, 8.25 \mathrm{mmol})$ and AIBN (0.47 mg, $0.003 \mathrm{mmol})$ were dissolved in 1,4-dioxane $(2 \mathrm{~mL})$ in a Schlenk flask. The solution was then freeze-pump-thawed four times and heated for $16 \mathrm{~h}$ at $70{ }^{\circ} \mathrm{C}$. The reaction was quenched by immersion of the ampoule in liquid nitrogen and the polymer was precipitated in hexane three times, followed by drying under vacuum (770 mg, 78\%). DP of P2VP was confirmed by ${ }^{1} \mathrm{H}$ NMR spectrum based on PLLA DP according to MALDI-TOF spectrometry. ${ }^{1} \mathrm{H}$ NMR $\left(400 \mathrm{MHz}, \mathrm{CDCl}_{3}\right): \delta(\mathrm{ppm})$ 8.41-8.07 (m, 503H, Ar from P2VP), 7.20$6.30\left(\mathrm{~m}, 1510 \mathrm{H}\right.$, Ar from P2VP), 5.16 (q, 94H, $\mathrm{C} \underline{\mathrm{H}}\left(\mathrm{CH}_{3}\right)$ from PLLA), 2.28-1.48 (1940H, m, $\mathrm{CH}\left(\mathrm{C}_{3}\right)$ from PLLA, $\underline{\mathrm{HC}}_{2} \underline{\mathrm{H}}_{2}$ from P2VP). GPC ( $n$-Bu $\mathrm{Bu}_{4} \mathrm{NBr} / \mathrm{THF}$, PS standard): $M_{\mathrm{n}}=63,245$ $\mathrm{g} \cdot \mathrm{mol}^{-1}, \bigoplus_{\mathrm{m}}=1.25$. $v_{\max }($ neat $) / \mathrm{cm}^{-1}: 3000-2880(\mathrm{C}-\mathrm{H}) ; 1755,1044(\mathrm{C}=\mathrm{O}) ; 1590,1585(\mathrm{Ar})$; 1472, 1433 (Ar-C-N); 1088 (C-O); 745 (Ar).

\section{Self-assembly procedures}

All solvent compositions are given as volume ratio $(v: v)$. All micelle length measurements were carried out on $\sim 200$ micelles.

Self-nucleation of PLLA47-b-PNIPAm267. $100 \mu \mathrm{L}$ of PLLA $47-b$-PNIPAm 267 solution $(10 \mathrm{mg} / \mathrm{mL}$ in DMSO) was added to a vial with an additional $100 \mu \mathrm{L}$ of DMSO followed by slow addition of $1800 \mu \mathrm{L}$ of EtOH resulting a polymer solution of $0.5 \mathrm{mg} / \mathrm{mL}$. The vial contents were aged at $23{ }^{\circ} \mathrm{C}$ for 5 days before TEM characterization. A large amount of non-spherical 
aggregates and polydisperse micelles was observed by TEM images (Figure S7d). To avoid the formation of spherical aggregates a heating-cooling method was employed. $100 \mu \mathrm{L}$ of PLLA47$b$-PNIPAm 267 solution $(10 \mathrm{mg} / \mathrm{mL}$ in DMSO) was added to a vial with $100 \mu \mathrm{L}$ DMSO and 1800 $\mu \mathrm{L}$ EtOH resulting a polymer solution of $0.5 \mathrm{mg} / \mathrm{mL}$. The vial was sealed and heated at $70{ }^{\circ} \mathrm{C}$ for $4 \mathrm{~h}$, followed by slowly cooling to $23{ }^{\circ} \mathrm{C}$. And the solution was carried on aging for $24 \mathrm{~h}$. The polydisperse fiber-like micelles formed were characterized by TEM.

Self-nucleation of PLLA 47- $\boldsymbol{b}$-P2VP 503 $100 \mu \mathrm{L}$ of PLLA $47-b-{ }^{2} 2 \mathrm{VP}_{503}$ solution $(10 \mathrm{mg} / \mathrm{mL}$ in DMSO) was added to a vial with $100 \mu \mathrm{L}$ DMSO and $1800 \mu \mathrm{L}$ EtOH resulting a polymer solution of $0.5 \mathrm{mg} / \mathrm{mL}$. The vial was sealed and heated at $70{ }^{\circ} \mathrm{C}$ for $4 \mathrm{~h}$, followed by slowly cooling to $23{ }^{\circ} \mathrm{C}$. And the solution was carried on aging for $24 \mathrm{~h}$. The formed polydisperse fiber-like micelles were characterized by TEM.

Preparation of seed micelles. All seed micelle solutions were prepared by sonication of the polydisperse micelle solutions from self-nucleation of polymer in selective solvents and characterized by TEM.

For PLLA $47-b$-PNIPAm 267 seeds $\left(L_{\mathrm{n}}=36 \mathrm{~nm}, L_{\mathrm{w}} / L_{\mathrm{n}}=1.10, \sigma / L_{\mathrm{n}}: 0.26\right)$ : sonication of PLLA47$b$-PNIPAm267 polydisperse micelles in DMSO/EtOH (1:9) was carried out for $2 \mathrm{~h}$ in a water sonication bath cooled with ice.

For PLLA $47-b$-PNIPAm 267 seeds $\left(L_{\mathrm{n}}=33 \mathrm{~nm}, L_{\mathrm{w}} / L_{\mathrm{n}}=1.13, \sigma / L_{\mathrm{n}}: 0.38\right)$ : sonication of PLLA $47^{-}$ $b$-PNIPAm 267 polydisperse micelles in DMSO/EtOH (1:9) was carried out for $2 \mathrm{~h}$ in a dry ice/acetone bath with a Ultrasonication probe.

For PLLA $47-b-\mathrm{P} 2 \mathrm{VP}_{503}$ seeds $\left(L_{\mathrm{n}}=29 \mathrm{~nm}, L_{\mathrm{w}} / L_{\mathrm{n}}=1.11, \sigma / L_{\mathrm{n}}: 0.34\right)$ : sonication of PLLA $47-b-$ PNIPAm 267 polydisperse micelles in DMSO/EtOH (1:9) was carried out for $2 \mathrm{~h}$ in a in a water sonication bath cooled with ice.

\section{Seeded-growth of PLLA47-b-PNIPAm267.}

For seeded-growth without $\mathrm{H}$-bond disruption reagents: $20 \mu \mathrm{L}$ (for $m_{\text {unimer }}: m_{\text {seed }} \leq 10.0$ ) or $10 \mu \mathrm{L}$ (for $m_{\text {unimer }}: m_{\text {seed }}>10.0$ ) of seed micelle solution $(0.5 \mathrm{mg} / \mathrm{mL}, \mathrm{DMSO}: \mathrm{EtOH}=1: 9$ ) was diluted in $400 \mu \mathrm{L}$ EtOH to which was added PLLA $47-b$-PNIPAm 267 unimer $(10 \mathrm{mg} / \mathrm{mL}$ in DMSO). The volumes of unimer added in were $2.5,5,10,7.5,10$ and $15 \mu \mathrm{L}$ corresponds to unimer-to-seed mass ratios of 2.5, 5, 10, 15, 20 and 30, respectively. And the resulting solution was then manually shaken for $10 \mathrm{~s}$ and aged for 5 days at $23{ }^{\circ} \mathrm{C}$ before TEM characterization. 
For seeded-growth with H-bond disruption reagent: $20 \mu \mathrm{L}$ (for $m_{\text {unimer }}: m_{\text {seed }} \leq 10.0$ ) or $10 \mu \mathrm{L}$ (for $\left.m_{\text {unimer }}: m_{\text {seed }}>10.0\right)$ of seed micelle solution $(0.5 \mathrm{mg} / \mathrm{mL}$, DMSO:EtOH $=1: 9)$ was diluted in $400 \mu \mathrm{L} \mathrm{TFE/EtOH} \mathrm{with} \mathrm{volume} \mathrm{ratio} \mathrm{of} \mathrm{3:97,} \mathrm{3:97,} \mathrm{5:95,} \mathrm{8:92,} \mathrm{10:90} \mathrm{and} \mathrm{15:85} \mathrm{corresponds}$ to unimer-to-seed mass ratios of $2.5,5,10,15,20$ and 30, respectively, and to which solution was added PLLA47- $b$-PNIPAm 267 unimer $(10 \mathrm{mg} / \mathrm{mL}$ in DMSO) with volumes of $2.5,5,10$, $7.5,10$ and $15 \mu \mathrm{L}$ respectively. And the resulting solution was then manually shaken for $10 \mathrm{~s}$ and aged for 5 days at $23{ }^{\circ} \mathrm{C}$ before TEM characterization.

\section{Seeded-growth of PLLA47-b-PNIPAm267 for kinetic studies.}

Same procedures were adopted with seeded-growth experiments of PLLA $47-b$-PNIPAm 267. After unimer addition, aliquots were taken after samples aged for $1 \mathrm{~d}, 3 \mathrm{~d}$, and $5 \mathrm{~d}$ for TEM characterization.

\section{Seeded-growth of PLLA47-b-PNIPAm267 for solvent effect studies.}

$10 \mu \mathrm{L}$ of seed micelle solution ( $0.5 \mathrm{mg} / \mathrm{mL}$, DMSO:EtOH $=1: 9)$ was diluted in $400 \mu \mathrm{L}$ solution of EtOH and selected solvents (MeOH, THF, Dioxane, DMF, DMSO, Acetone and TFE) individually with volume ratio of 0.5:9.5, 1:9 and 1.5:8.5, respectively. Similar procedures of unimer addition were adopted with seeded-growth experiments of PLLA47- $b$-PNIPAm 267 . And the resulting solution was then manually shaken for $10 \mathrm{~s}$ and aged for 5 days at $23{ }^{\circ} \mathrm{C}$ before TEM characterization.

\section{Preparation of samples for SAXS analysis.}

To obtain quality data from SAXS experiments, micelles were prepared at higher concentrations compare with the method mentioned above. Polydisperse micelles of PLLA47$b$-PNIPAm 267 was prepared at a polymer concentration of $1 \mathrm{mg} / \mathrm{mL}$ by adding $200 \mu \mathrm{L}$ of unimer solution $(10 \mathrm{mg} / \mathrm{mL}$ in THF) to $1800 \mu \mathrm{L}$ of EtOH. THF was employed as the common solvent in this preparation due to the large volatility compared with that of DMSO, which allowing the micelle solutions to be concentrated by applying nitrogen flow and resulting micelles suspended in TFE/EtOH. The solution was heated at $70{ }^{\circ} \mathrm{C}$ for 4 hours and slowly cool to $23{ }^{\circ} \mathrm{C}$. The solution was further aged for 5 days and then characterized by TEM. The formed polydisperse micelles were sonicated at $-78{ }^{\circ} \mathrm{C}$ in a dry ice/acetone bath for $1 \mathrm{~h}$ with a Ultrasonicate probe. TEM images showed that the seed micelles had a $L_{\mathrm{n}}$ (and $L_{\mathrm{w}} / L_{\mathrm{n}}$ ) of 36 (1.05) nm. $260 \mu \mathrm{L}$ seed solution was diluted in $1 \mathrm{~mL}$ TFE/EtOH (1:9). To the seed solution, $94 \mu \mathrm{L}$ of unimer $(50 \mathrm{mg} / \mathrm{mL}$ in THF) was added and the solution was manually shaken for $10 \mathrm{~s}$. 
The final solution had a polymer concentration of $4.6 \mathrm{mg} / \mathrm{mL}$. After ageing for $24 \mathrm{~h}$, the micelle was determined a $L_{\mathrm{n}}\left(\right.$ and $\left.L_{\mathrm{w}} / L_{\mathrm{n}}\right)$ value of 1040 (1.04) by TEM. The sample with a concentration of $4 \mathrm{mg} / \mathrm{mL}$ was prepared by adding $50 \mu \mathrm{L}$ TFE/EtOH (1:9) to the micelle solution $(100 \mu \mathrm{L})$. The rest micelle solution was concentrated by applying nitrogen flow to $\sim 120 \mu \mathrm{L}$. The concentrated micelle solution had a concentration of $30 \mathrm{mg} / \mathrm{mL}$. Samples with $20 \mathrm{mg} / \mathrm{mL}$ was prepared by adding $25 \mathrm{uL} \mathrm{TFE/EtOH} \mathrm{(1:9)} \mathrm{to} \mathrm{the} \mathrm{concentrated} \mathrm{micelle} \mathrm{solution} \mathrm{(50 \mu L).} \mathrm{The}$ micelle solution prepared for SAXS had been used in other characterizations (AFM, SAED and PXRD).

\section{Seeded-growth of PLLA47-b-P2VP503.}

Analogous procedures were adopted to those used for the seeded-growth experiments with PLLA47- $b$-PNIPAm 267 in both EtOH and TFE/EtOH. After the unimer addition, samples were manually shaken for $10 \mathrm{~s}$ and aged for 5 days at $23{ }^{\circ} \mathrm{C}$ before TEM characterization.

\section{Preparation of pentablock co-micelles}

For central block 1 micelles: $20 \mu \mathrm{L}$ of PLLA $47-b$-PNIPAm 267 seed micelle solution $\left(L_{\mathrm{n}}=33 \mathrm{~nm}\right.$, $0.5 \mathrm{mg} / \mathrm{mL}, \mathrm{DMSO} / \mathrm{EtOH}=1: 9)$ was diluted in $400 \mu \mathrm{L}$ TFE/EtOH (1:9) to which was added PLLA $_{47}-b$-PNIPAm 267 unimer $(9 \mu \mathrm{L}, 10 \mathrm{mg} / \mathrm{mL}$ in DMSO). And the resulting solution was then manually shaken for $10 \mathrm{~s}$ and aged for 3 days at $23{ }^{\circ} \mathrm{C}$ before TEM characterization.

For triblock 1 co-micelles: PLLA $47-b-\mathrm{P}_{2} \mathrm{VP}_{503}$ unimer $(3 \mathrm{uL}, 10 \mathrm{mg} / \mathrm{mL}$ in DMSO) was then added to the central block 1 micelle solution $(200 \mathrm{uL})$. The resulting solution was then manually shaken for $10 \mathrm{~s}$ and aged for 3 days at $23{ }^{\circ} \mathrm{C}$ before TEM characterization.

For pentablock 1 co-micelles: PLLA $47-b$-PNIPAm 267 unimer $(3 \mu \mathrm{L}, 10 \mathrm{mg} / \mathrm{mL}$ in DMSO) was added to triblock 1 co-micelle solution (100 uL) (see above). The resulting solution was then manually shaken for $10 \mathrm{~s}$ and aged for 3 days at $23{ }^{\circ} \mathrm{C}$ before TEM characterization.

For central block 2 micelles: $20 \mu \mathrm{L}$ of PLLA $47-b-\mathrm{P} 2 \mathrm{VP}_{503}$ seed micelle solution $\left(L_{\mathrm{n}}=29 \mathrm{~nm}\right.$, $0.5 \mathrm{mg} / \mathrm{mL}, \mathrm{DMSO} / \mathrm{EtOH}=1: 9)$ was diluted in $400 \mu \mathrm{L}$ TFE/EtOH (1:9) to which was added $\mathrm{PLLA}_{47}-b-\mathrm{P} 2 \mathrm{VP}_{503}$ unimer $(9 \mu \mathrm{L}, 10 \mathrm{mg} / \mathrm{mL}$ in DMSO). And the resulting solution was then manually shaken for $10 \mathrm{~s}$ and aged for 3 days at $23{ }^{\circ} \mathrm{C}$ before TEM characterization.

For triblock 2 co-micelles: PLLA47- $b$-PNIPAm 267 unimer $(5 \mathrm{uL}, 10 \mathrm{mg} / \mathrm{mL}$ in DMSO) was then added to the central block 2 micelle solution $(200 \mathrm{uL}$ ) (see above). The resulting solution was then manually shaken for $10 \mathrm{~s}$ and aged for 3 days at $23{ }^{\circ} \mathrm{C}$ before TEM characterization. 
For pentablock 2 co-micelles: PLLA $47-b$-P2 $\mathrm{VP}_{503}$ unimer $(5 \mu \mathrm{L}, 10 \mathrm{mg} / \mathrm{mL}$ in DMSO) was added to the triblock 2 co-micelle solution (100 uL) (see above). The resulting solution was then manually shaken for $10 \mathrm{~s}$ and aged for 3 days at $23{ }^{\circ} \mathrm{C}$ before TEM characterization.

\section{Discussion on defects in pentablock comicelles.}

Triblock co-micelles and pentablock co-micelles were successfully prepared by alternating seeded-growth of PLLA $47-b$-PNIPAm 267 and PLLA $47-b-$ P2VP $_{503}$ unimers. One of the features of these block co-micelles was that the grown segments were not equivalent in length as would be expected for a controlled living CDSA process (Figure 9). This is proposed caused by the different addition rates of BCPs with different corona-forming block lengths as previously reported..$^{4-6}$ In a typical experiment of preparing triblock co-micelles, the growth of PLLA $47-b$ PNIPAm $_{267}$ unimer from PLLA $47-b-\mathrm{P}_{2} \mathrm{VP}_{503}$ seed micelles is suggested to be a slow step as a consequence of unfavourable corona-corona interactions between PNIPAm $\left(\delta=24.8 \mathrm{MPa}^{1 / 2}\right)$ and P2VP $\left(\delta=20.8 \mathrm{MPa}^{1 / 2}\right)$. Once the first unimer from PLLA $47-b$-PNIPAm 267 has been added in this slow step, further unimers can deposit more rapidly. This might result in different lengths in the newly grown outer segments. In the preparation of pentablock co-micelles, the nonequivalent growth would be amplified by the same reason of switching to a different unimers.

\section{Abbreviation}

DCM: dichloromethane; TFE: trifluoroethanol; MeOH: methanol; EtOH: ethanol; DMF: N,NDimethylformamide; DMSO: Dimethyl sulfoxide; THF: tetrahydrofuran; Dioxane: 1,4dioxane; CDSA: crystallization-driven self-assembly; $\sigma$ : standard deviation. 


\section{Supplementary figures}
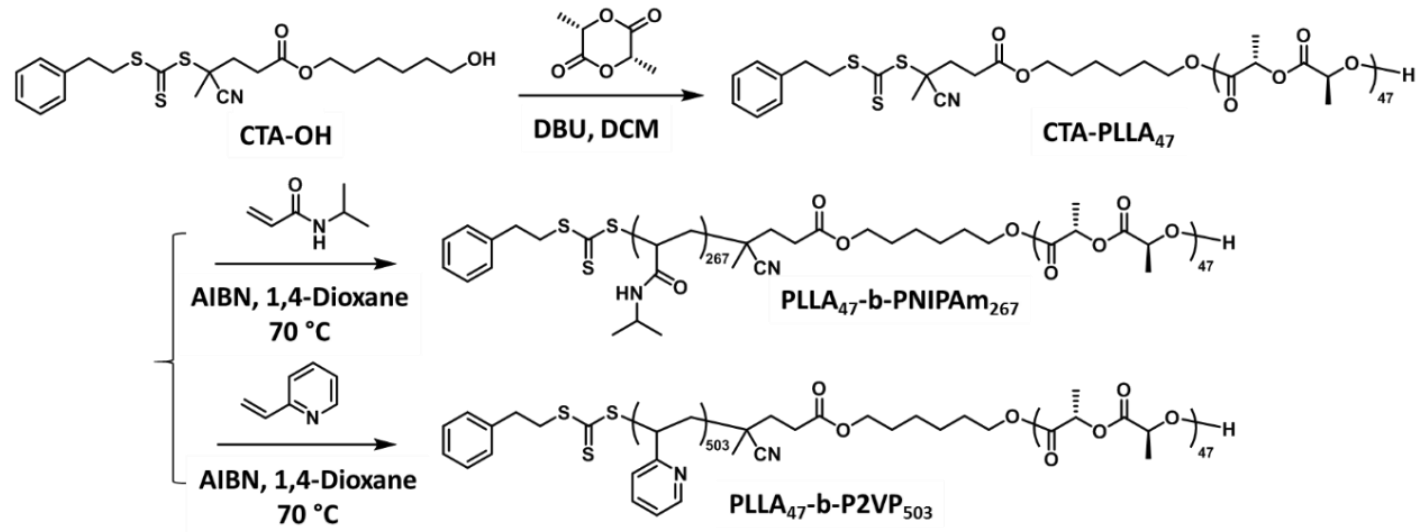

Scheme S1. Synthesis of PLLA $47-b$-PNIPAm 267 and PLLA $47-b-$ P2 $_{403}$ diblock copolymers

Table S1. Molar mass characterization of the polymers prepared.

\begin{tabular}{|c|c|c|c|c|c|}
\hline Polymer & $\begin{array}{c}M_{\mathrm{w}} \\
\left(\mathrm{g} \cdot \mathrm{mol}^{-1}\right)^{\mathrm{a}}\end{array}$ & $\begin{array}{c}M_{\mathrm{n}} \\
\left(\mathrm{kg} \cdot \mathrm{mol}^{-1}\right)^{\mathrm{b}}\end{array}$ & $\begin{array}{c}M_{\mathrm{n}} \\
\left(\mathrm{kg} \cdot \mathrm{mol}^{-1}\right)^{\mathrm{c}}\end{array}$ & $\boldsymbol{D}_{\mathrm{M}}{ }^{\mathrm{c}}$ & $\begin{array}{c}\text { Block ratio } \\
\text { (Core:Corona) }\end{array}$ \\
\hline CTA-PLLA $_{47}$ & 7232 & 7.6 & 10.2 & 1.09 & - \\
\hline PLLA $_{47}-b-$ PNIPAm $_{267}$ & - & 37.4 & $180^{d}$ & 1.11 & $1: 5$ \\
\hline $\mathrm{PLLA}_{47}-b-\mathrm{P} 2 \mathrm{VP}_{503}$ & - & 59.7 & 63.2 & 1.25 & $1: 10$ \\
\hline
\end{tabular}

a determined by MALDI;

${ }^{\mathrm{b}}$ block ratio (according to degree of polymerization (DP)) determined by ${ }^{1} \mathrm{H}$ NMR spectroscopy;

c determined by GPC relative to polystyrene (PS) standards in $n$ $\mathrm{Bu}_{4} \mathrm{NBr} / \mathrm{THF}$.

d The molar mass estimated by GPC is much larger than that determined by ${ }^{1} \mathrm{H}$ NMR integration. We attribute this to exclusion interactions between the high polar polymer and the low-polarity styrene/divinylbenzene column. Such effects have been previously noted for highly polar polymers ${ }^{7}$ 


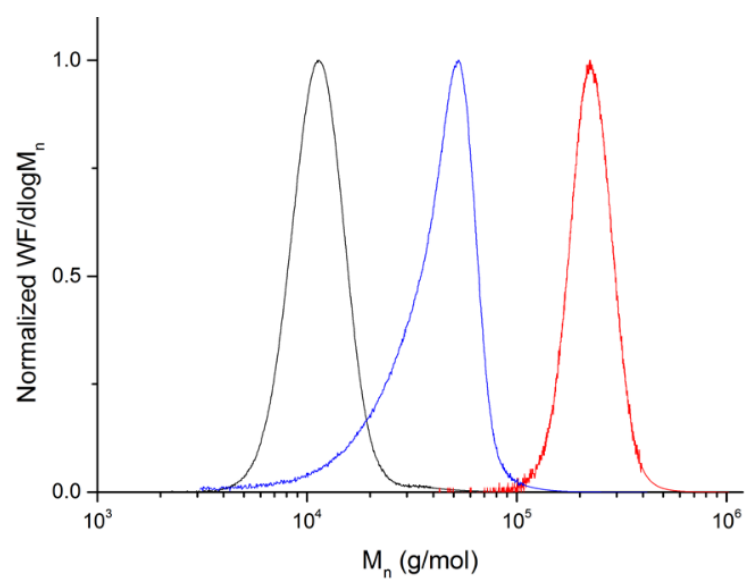

Figure S1. GPC chromatographs (refractive index trace) in n-Bu $\mathrm{Bu}_{4} \mathrm{NBr} / \mathrm{THF}$ of CTA-PLLA 47 (black, $\nexists_{\mathrm{m}}$ $=1.09), \mathrm{PLLA}_{47}-b-\mathrm{P}_{2 \mathrm{VP}} \mathrm{V}_{53}\left(\right.$ blue, $\left.\bigoplus_{\mathrm{m}}=1.25\right), \mathrm{PLLA}_{47}-b-\mathrm{PNIPAm}_{267}\left(\right.$ red, $\left.\bigoplus_{\mathrm{m}}=1.11\right)$.

(a)

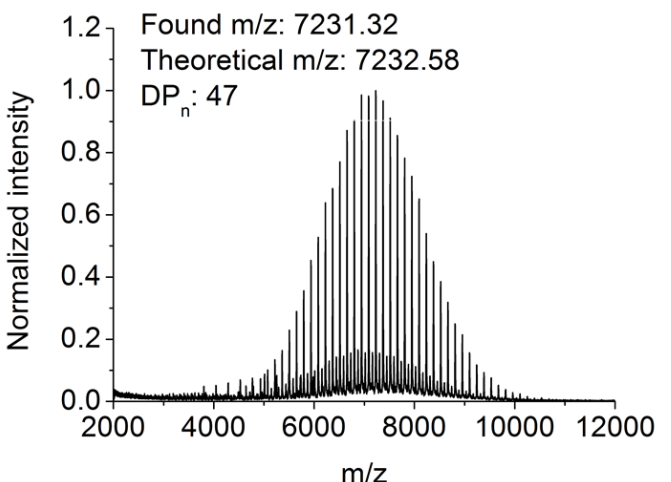

(b)

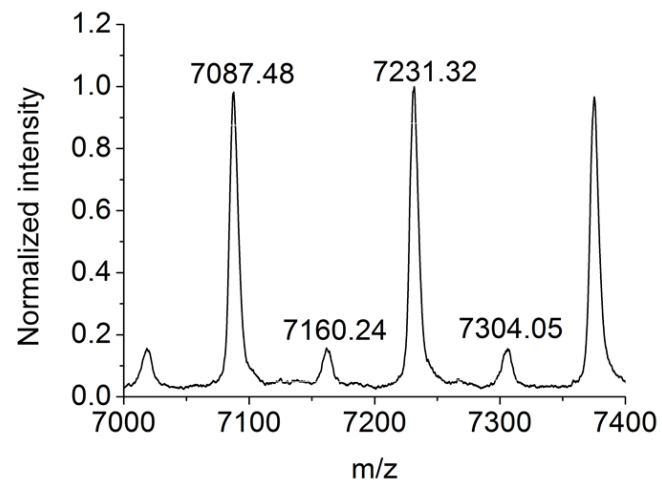

(c)

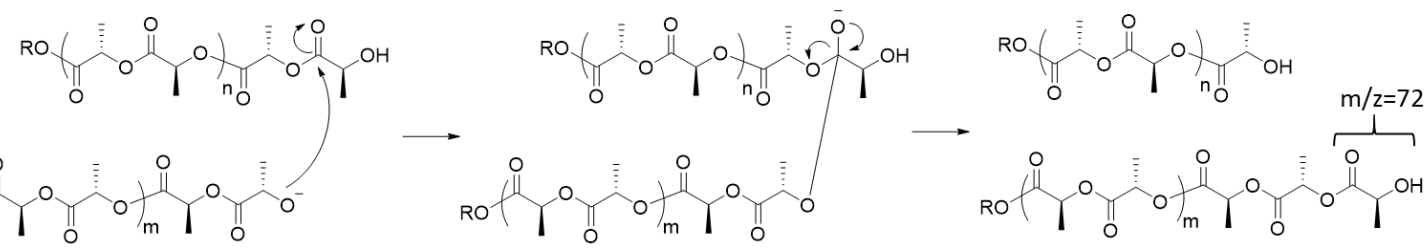

Figure S2. (a) MALDI-TOF MS of CTA-PLLA47; (b) Zoom-in MALDI-TOF MS of CTA-PLLA47;. (c) Mechanism of transesterification in $L$-lactide polymerization, which corresponds to the minor population possessing a $\mathrm{m} / \mathrm{z}$ difference of 72 compared with major population in MALDI-TOF MS. 
(a)
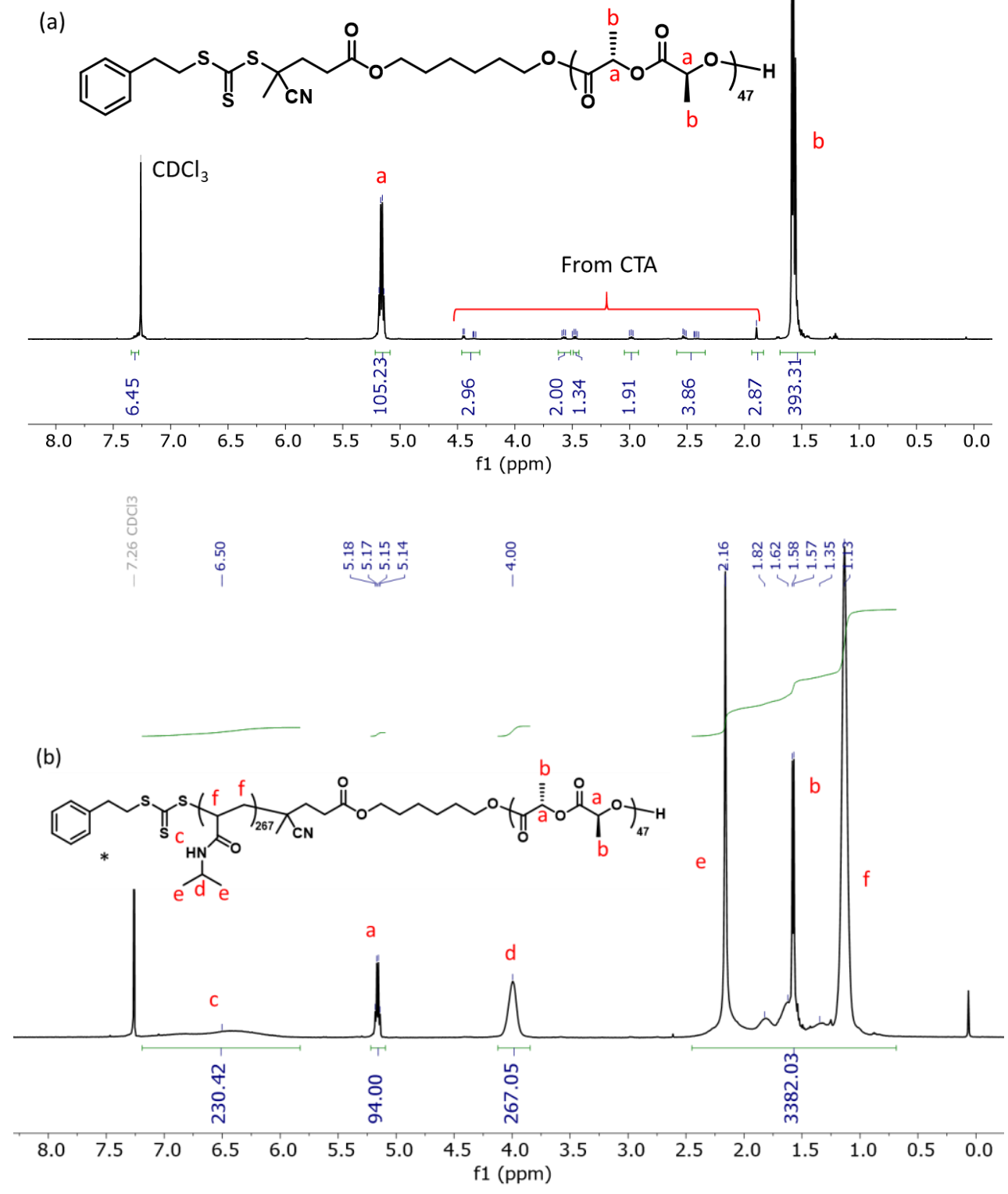

(b)

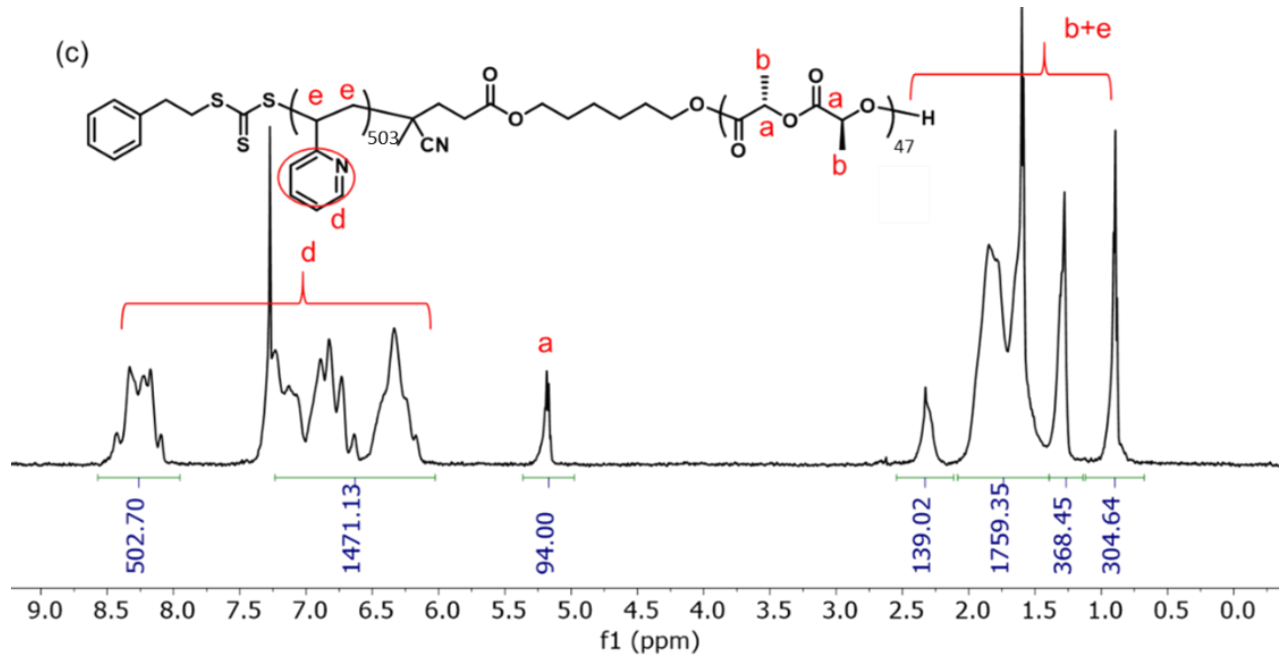

Figure S3. ${ }^{1} \mathrm{H}$ NMR (400 MHz, $\mathrm{CDCl}_{3}$ ) spectra of (a) CTA-PLLA 47 ; (b) PLLA $47-b$-PNIPAm 267 and (c) $\mathrm{PLLA}_{47}-b-\mathrm{P} 2 \mathrm{VP}_{503}$. 


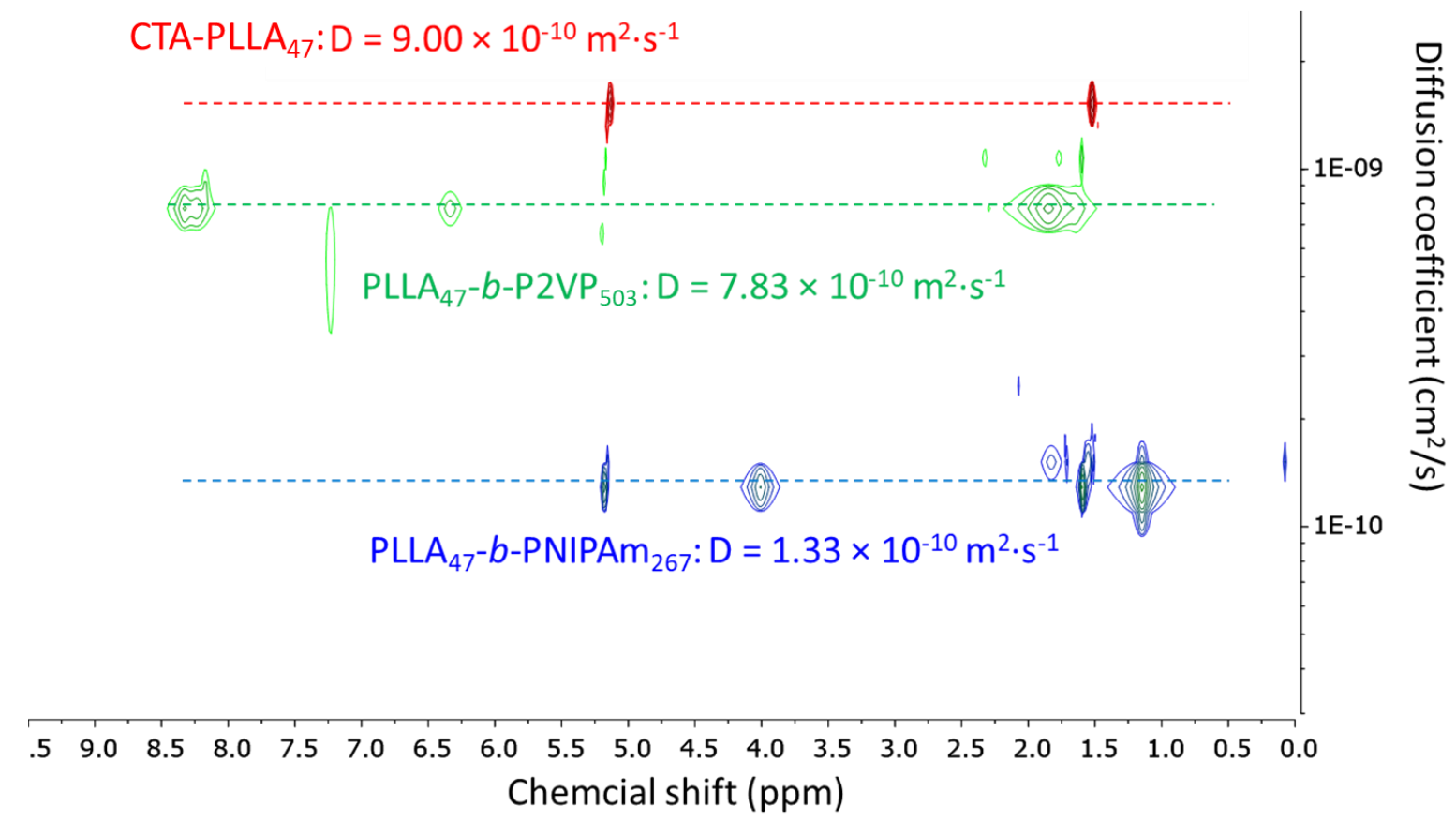

Figure S4. ${ }^{1} \mathrm{H}$ DOSY NMR $\left(500 \mathrm{MHz}, \mathrm{CDCl}_{3}\right)$ spectra of CTA-PLLA 47 (red), PLLA $47-b-\mathrm{P}_{2} \mathrm{VP}_{503}$ (green) and PLLA $47-b$-PNIPAm 267 (blue).

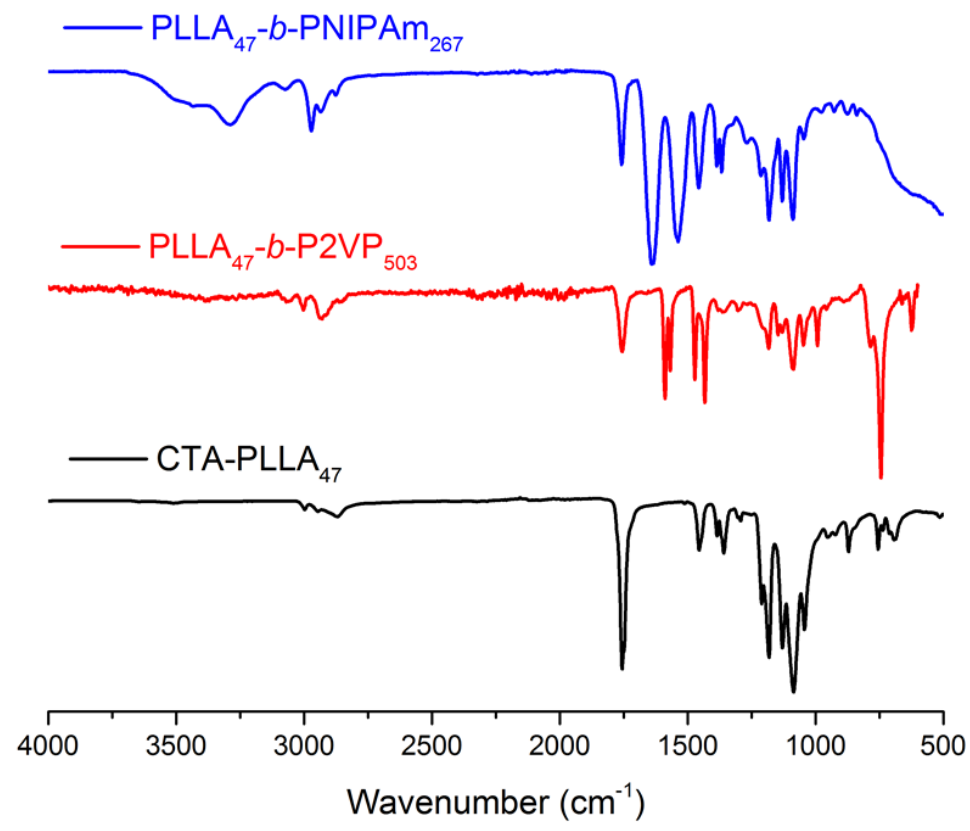

Figure S5. FT-IR characterization of PLLA-containing polymers. 


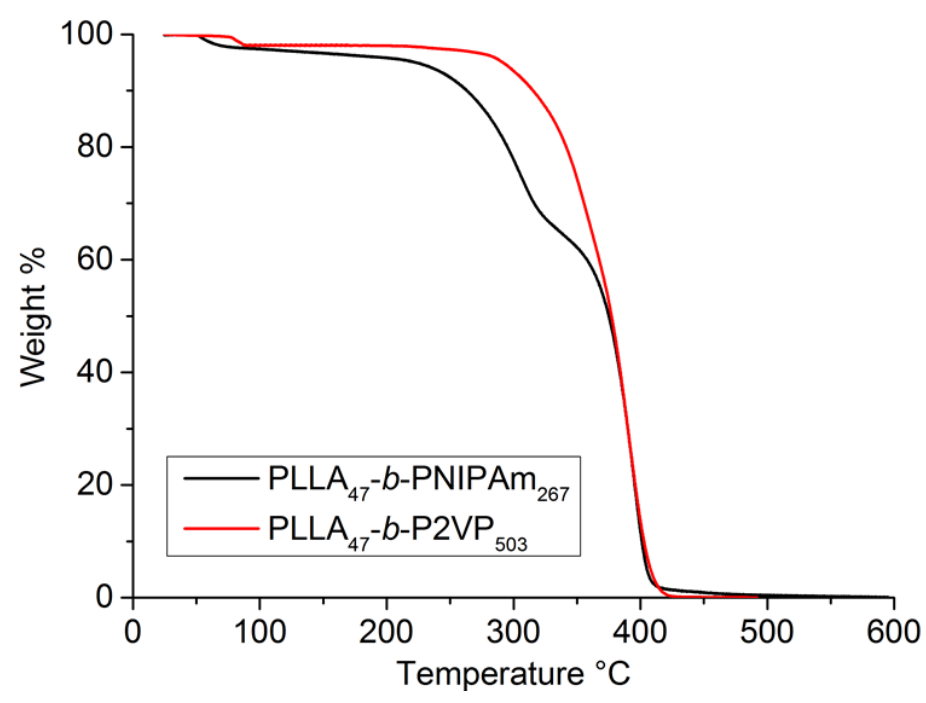

Figure S6. Thermogravimetric analysis (TGA) for PLLA $47-b-$ PNIPAm $_{267}$ (black) and PLLA $47-b-$ $\mathrm{P}^{2 \mathrm{VP}_{503}}$ (red). TGA was performed at a scan rate of $10{ }^{\circ} \mathrm{C} / \mathrm{min}$ under nitrogen.

(a)

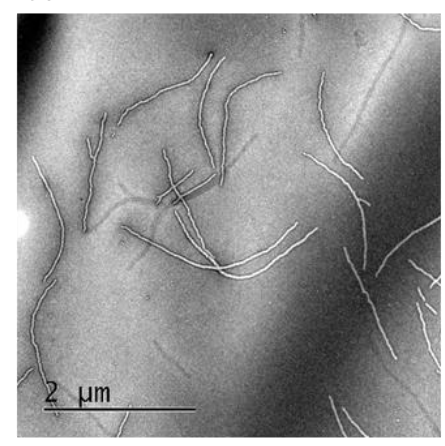

(d) (b)

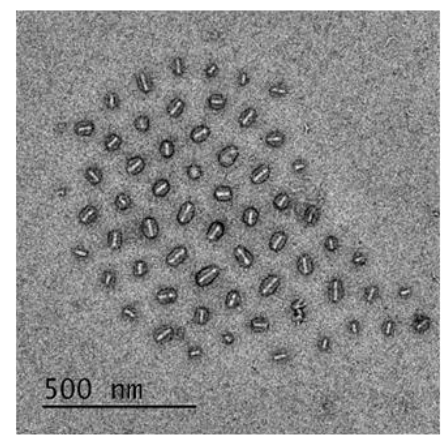

(c)

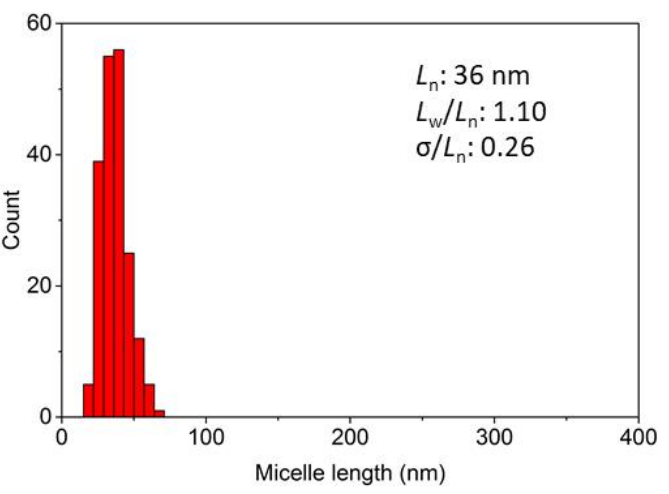

Figure S7. (a) TEM images of polydisperse PLLA $_{47}-b$-PNIPAm 267 micelles prepared by heating the polymer in DMSO/EtOH (1:9) at a concentration of $0.5 \mathrm{mg} / \mathrm{mL}$ at $70{ }^{\circ} \mathrm{C}$ for $2 \mathrm{~h}$ followed by slow cooling down to $23^{\circ} \mathrm{C}$; (b) TEM images of seeds prepared by sonication of polydisperse micelles at $0{ }^{\circ} \mathrm{C}$ for $2 \mathrm{~h}$ in a sonic cleaning bath; (c) contour length histogram of measured seeds length, $L_{\mathrm{n}}=36$ $\mathrm{nm}, L_{\mathrm{w}} / L_{\mathrm{n}}=1.10, \sigma / L_{\mathrm{n}}: 0.26$. TEM samples were stained with a $2 \mathrm{wt} \%$ solution of uranyl acetate in EtOH; (d) TEM images of polydisperse PLLA $47-b$-PNIPAm 267 micelles prepared by addition of unimers in DMSO to ethanol and aging at $23{ }^{\circ} \mathrm{C}$ for 5 days. A significant amount of non-spherical aggregates was observed. 
(a) $m_{\text {unimer }}: m_{\text {seed }}=2.5$

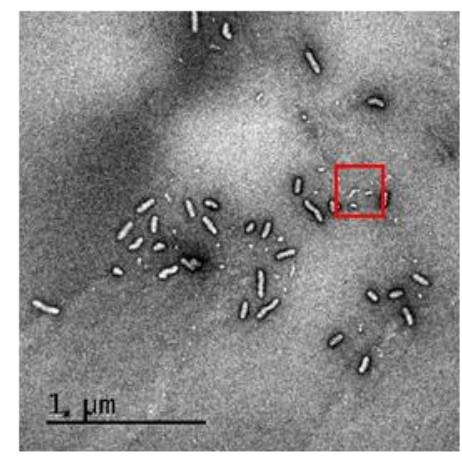

(d) $m_{\text {unimer }}: m_{\text {seed }}=15.0$

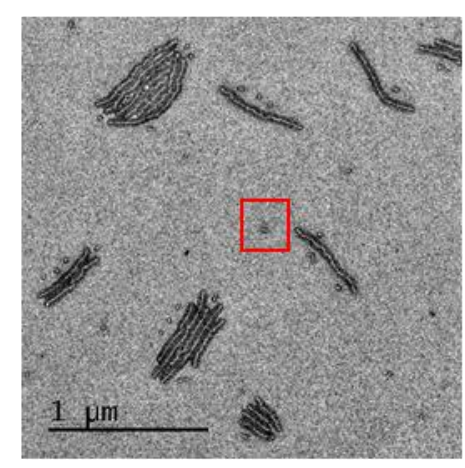

(b)

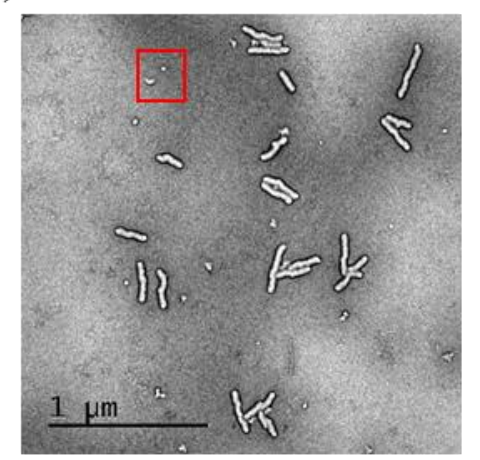

(e) $m_{\text {unimer }}: m_{\text {seed }}=20.0$

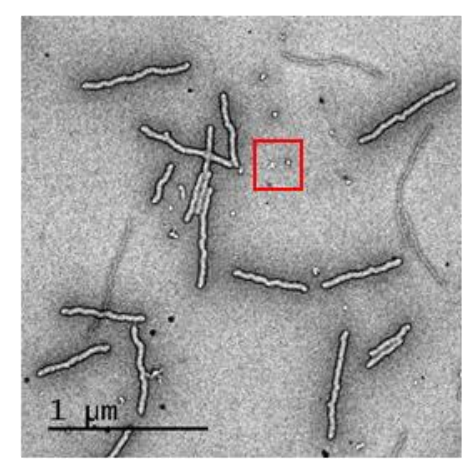

(c) $\quad m_{\text {unimer }}: m_{\text {seed }}=10.0$

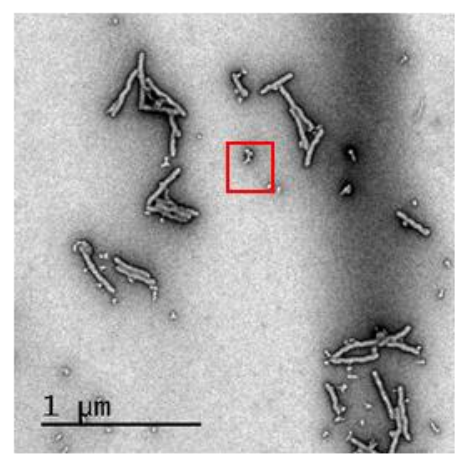

(f)

$$
m_{\text {unimer }}: m_{\text {seed }}=30.0
$$

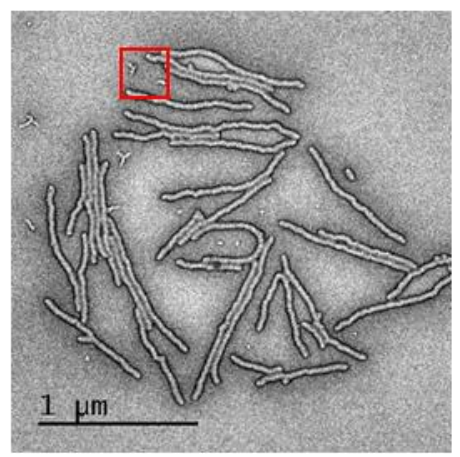

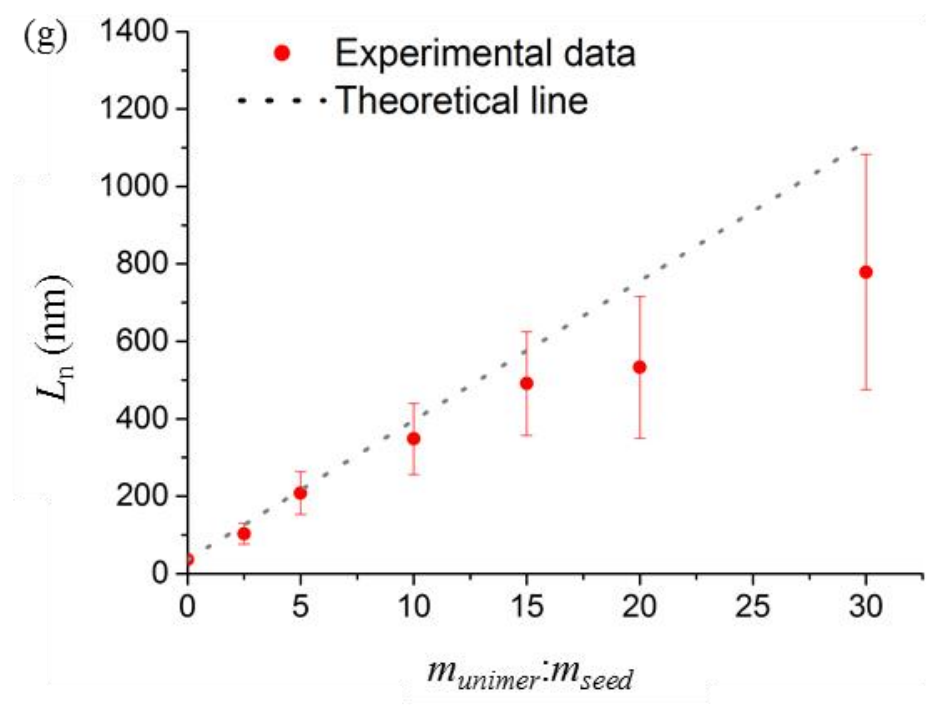

(h)

\begin{tabular}{cccc}
\hline $\begin{array}{c}m_{\text {unimer }} \\
: m_{\text {seed }}\end{array}$ & $\begin{array}{c}L_{\mathrm{n}} \\
(\mathrm{nm})\end{array}$ & $L_{\mathrm{w}} / L_{\mathrm{n}}$ & $\sigma / L_{\mathrm{n}}$ \\
\hline 2.5 & 103 & 1.06 & 0.25 \\
5.0 & 208 & 1.08 & 0.28 \\
10.0 & 348 & 1.06 & 0.26 \\
15.0 & 491 & 1.07 & 0.26 \\
20.0 & 533 & 1.12 & 0.30 \\
30.0 & 779 & 1.15 & 0.35 \\
\hline
\end{tabular}

Figure S8. TEM images of samples (aging for 5 days) of elongated PLLA $47-b$-PNIPAm 267 micelles prepared by seeded growth from seed micelles $\left(L_{\mathrm{n}}=36 \mathrm{~nm}, L_{\mathrm{w}} / L_{\mathrm{n}}=1.10, \sigma / L_{\mathrm{n}}: 0.26\right)$ in EtOH after addition of unimers (in DMSO) with unimer-to-seed mass ratios of (a) 2.5, (b) 5.0, (c) 10.0, (d) 15.0, (e) 20.0 and (f) 30.0; (g) plot of micelle number average length verse unimer-to-seed ratios (the error bars represent the standard deviation); (h) measured length data summary. Red rectangles highlight non-spherical particles. TEM samples were stained with a $2 \mathrm{wt} \%$ solution of uranyl acetate in EtOH. 

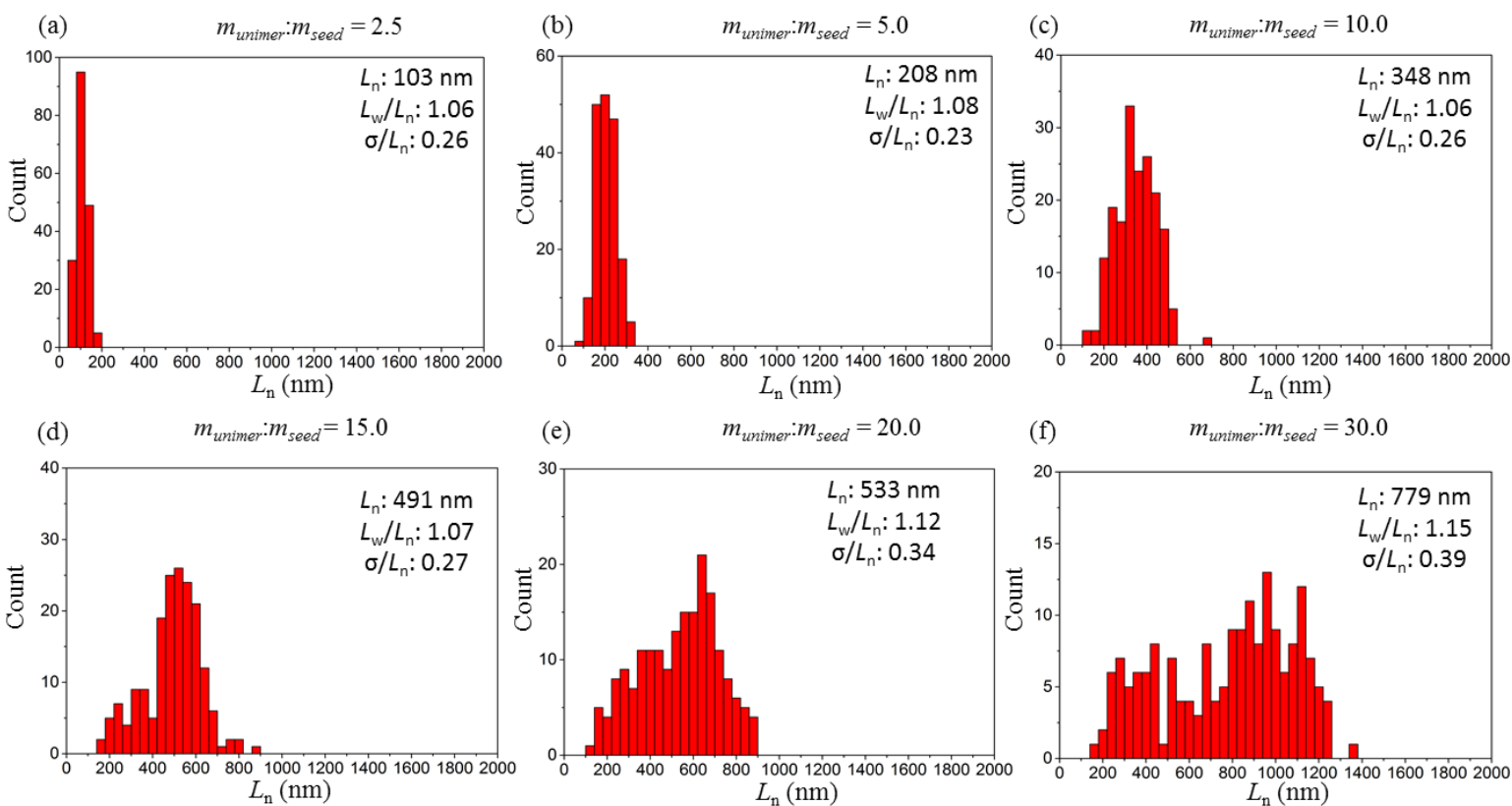

Figure S9. Contour length histogram of 5 days aged elongated PLLA $47-b$-PNIPAm 267 micelles prepared by seeded growth off seed micelles $\left(L_{\mathrm{n}}=36 \mathrm{~nm}, L_{\mathrm{w}} / L_{\mathrm{n}}=1.10, \sigma / L_{\mathrm{n}}: 0.26\right)$ in EtOH after the addition of unimers (in DMSO) with unimer-to-seed mass ratios of (a) 2.5, (b) 5.0, (c) 10.0, (d) 15.0, (e) 20.0 and (f) 30.0 .

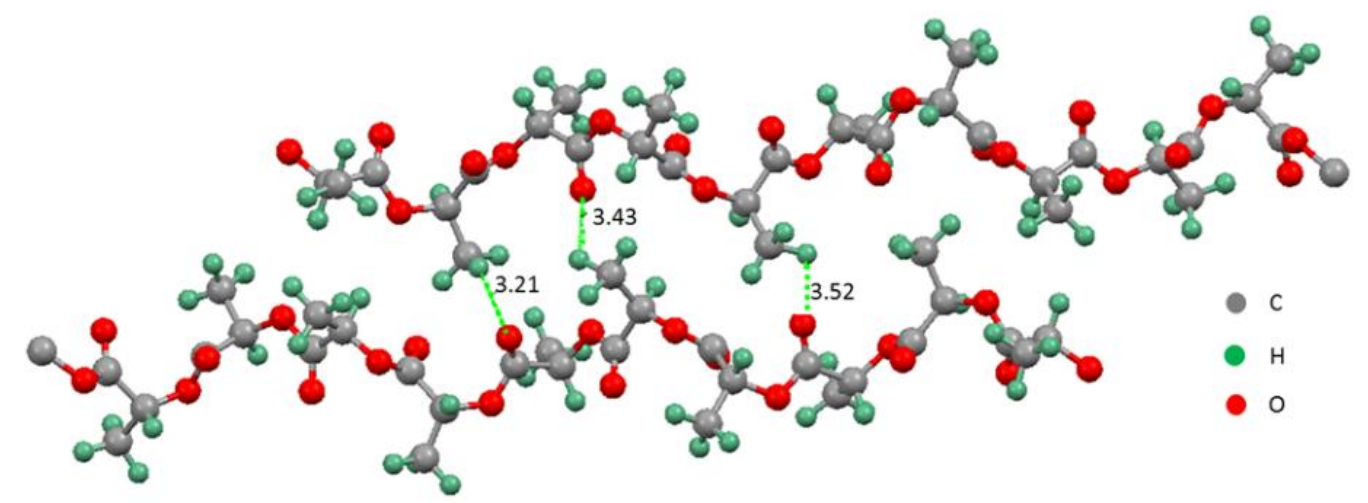

Figure S10. Measurement of $\mathrm{O} \cdots \mathrm{H}$ distance between two adjacent chains in a model of PLLA $\alpha$-form crystal cell unit. 

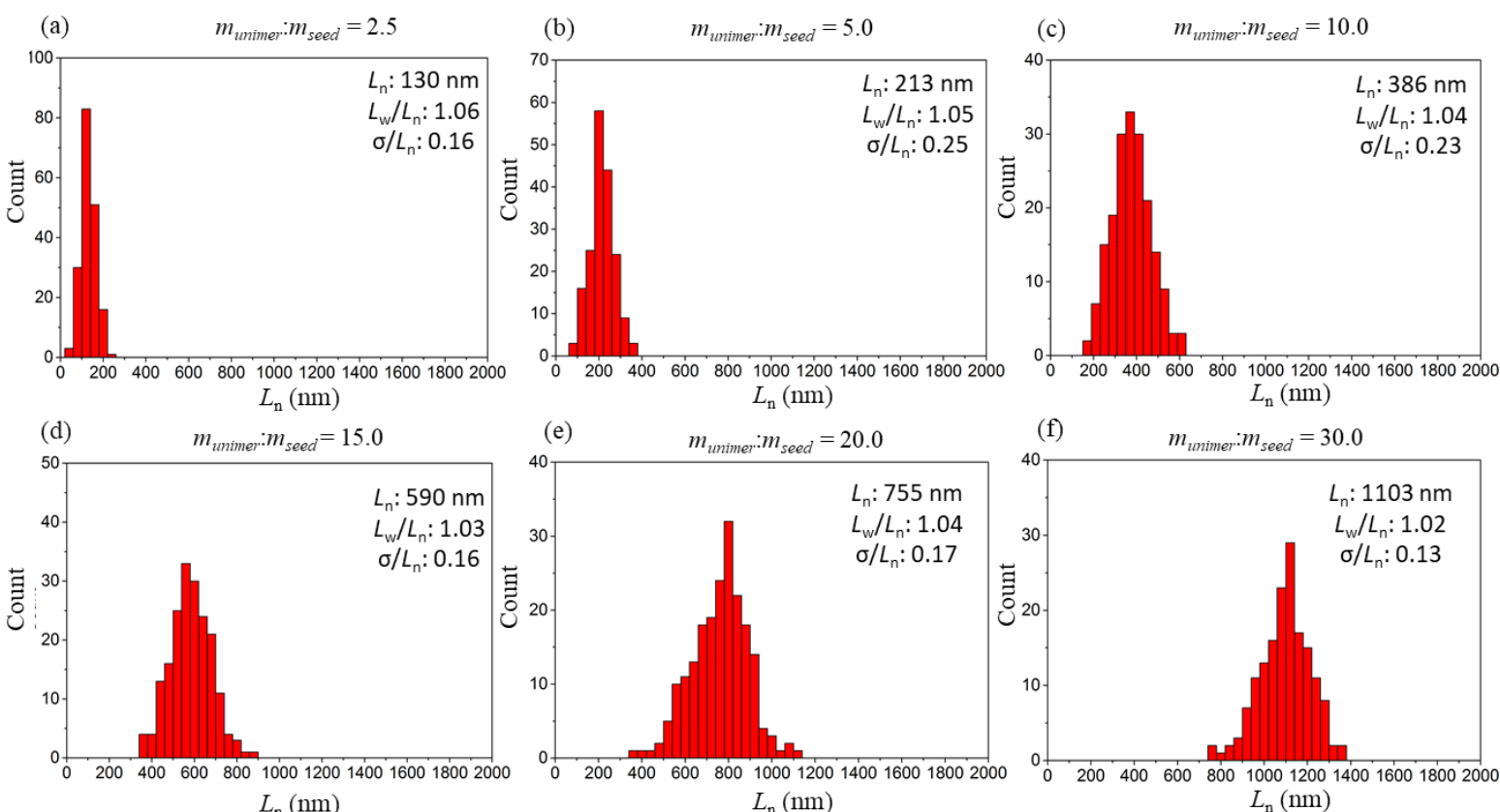

\begin{tabular}{ccccc}
\hline g) $\begin{array}{cccc}m_{\text {unimer }} \\
: m_{\text {seed }}\end{array}$ & $\begin{array}{c}v_{\text {TFE }} \\
v_{\text {ETOH }}\end{array}$ & $\begin{array}{c}L_{\mathrm{n}} \\
(\mathrm{nm})\end{array}$ & $L_{\mathrm{w}} / L_{\mathrm{n}}$ & $\sigma / L_{\mathrm{n}}$ \\
\hline 2.5 & $3: 97$ & 130 & 1.09 & 0.16 \\
\hline 5.0 & $3: 97$ & 213 & 1.06 & 0.25 \\
10.0 & $5: 95$ & 386 & 1.05 & 0.23 \\
15.0 & $8: 92$ & 590 & 1.03 & 0.16 \\
\hline 20.0 & $10: 90$ & 755 & 1.03 & 0.17 \\
\hline 30.0 & $15: 85$ & 1103 & 1.02 & 0.13 \\
\hline
\end{tabular}

Figure S11. Contour length histogram of 5 days aged uniform PLLA $47-b$-PNIPAm 267 micelles prepared by seeded growth off seed micelles $\left(L_{\mathrm{n}}=36 \mathrm{~nm}, L_{\mathrm{w}} / L_{\mathrm{n}}=1.10, \sigma / L_{\mathrm{n}}: 0.26\right)$ in TFE/EtOH with volume ratios of (a) 3:97, (b) 3:97, (c) 5:95, (d) 8:92, (e) 10:90 and (f) 15:85 after the addition of unimers (in DMSO) with unimer-to-seed mass ratios of (a) 2.5, (b) 5.0, (c) 10.0, (d) 15.0, (e) 20.0 and (f) 30.0, respectively; (g) measured length data summary. 


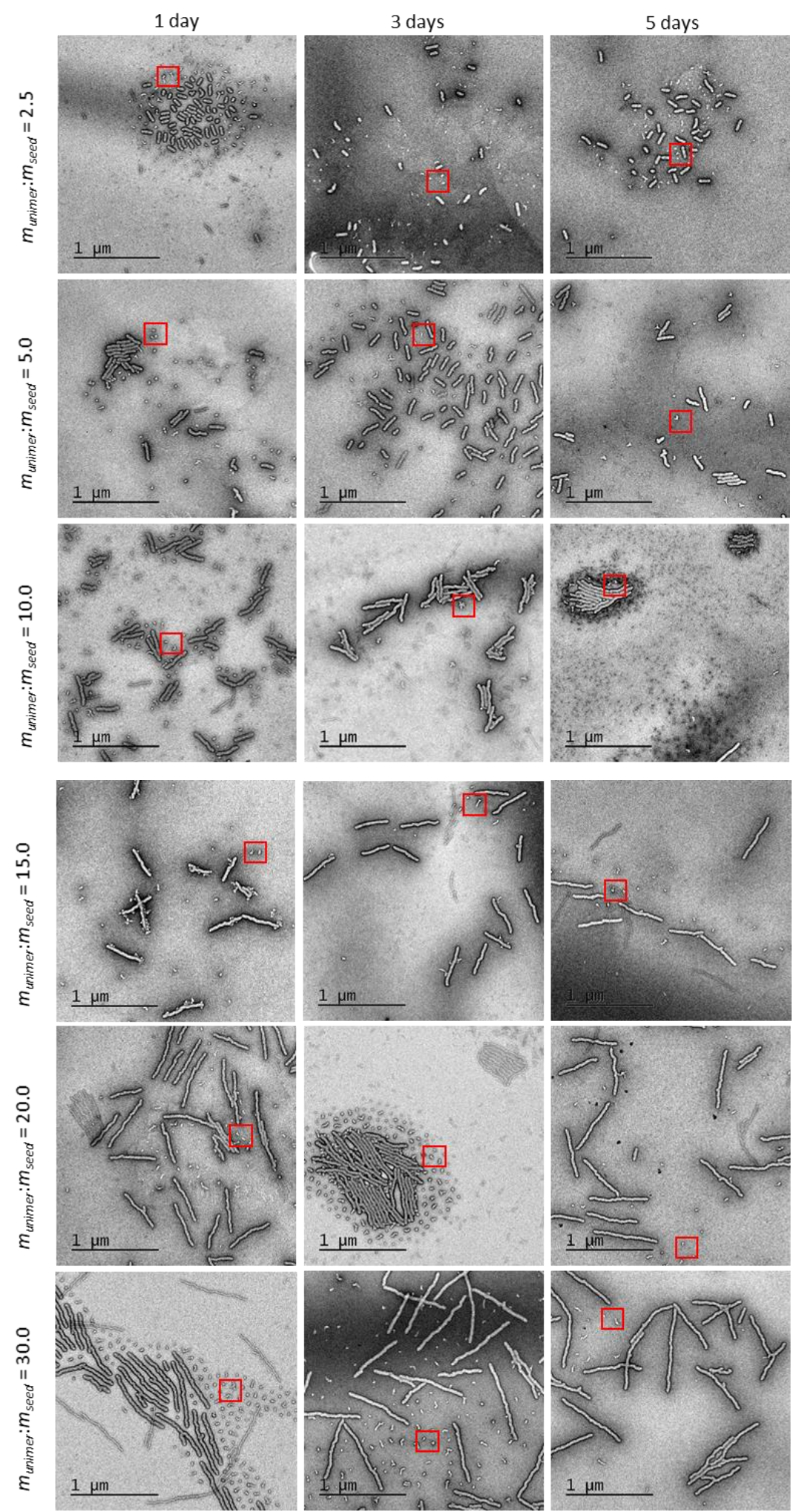

Figure S12. TEM images of elongated PLLA $_{47}-b$-PNIPAm 267 micelles in kinetic studies prepared by seeded growth off seed micelles $\left(L_{\mathrm{n}}=36 \mathrm{~nm}, L_{\mathrm{w}} / L_{\mathrm{n}}=1.10, \sigma / L_{\mathrm{n}}: 0.26\right)$ in EtOH after addition of unimers (in DMSO) with unimer-to-seed mass ratios of 2.5, 5.0, 10.0, 15.0, 20.0 and 30.0; Red rectangles highlight non-spherical particles. TEM samples were stained with a $2 \mathrm{wt} \%$ solution of uranyl acetate in $\mathrm{EtOH}$. 

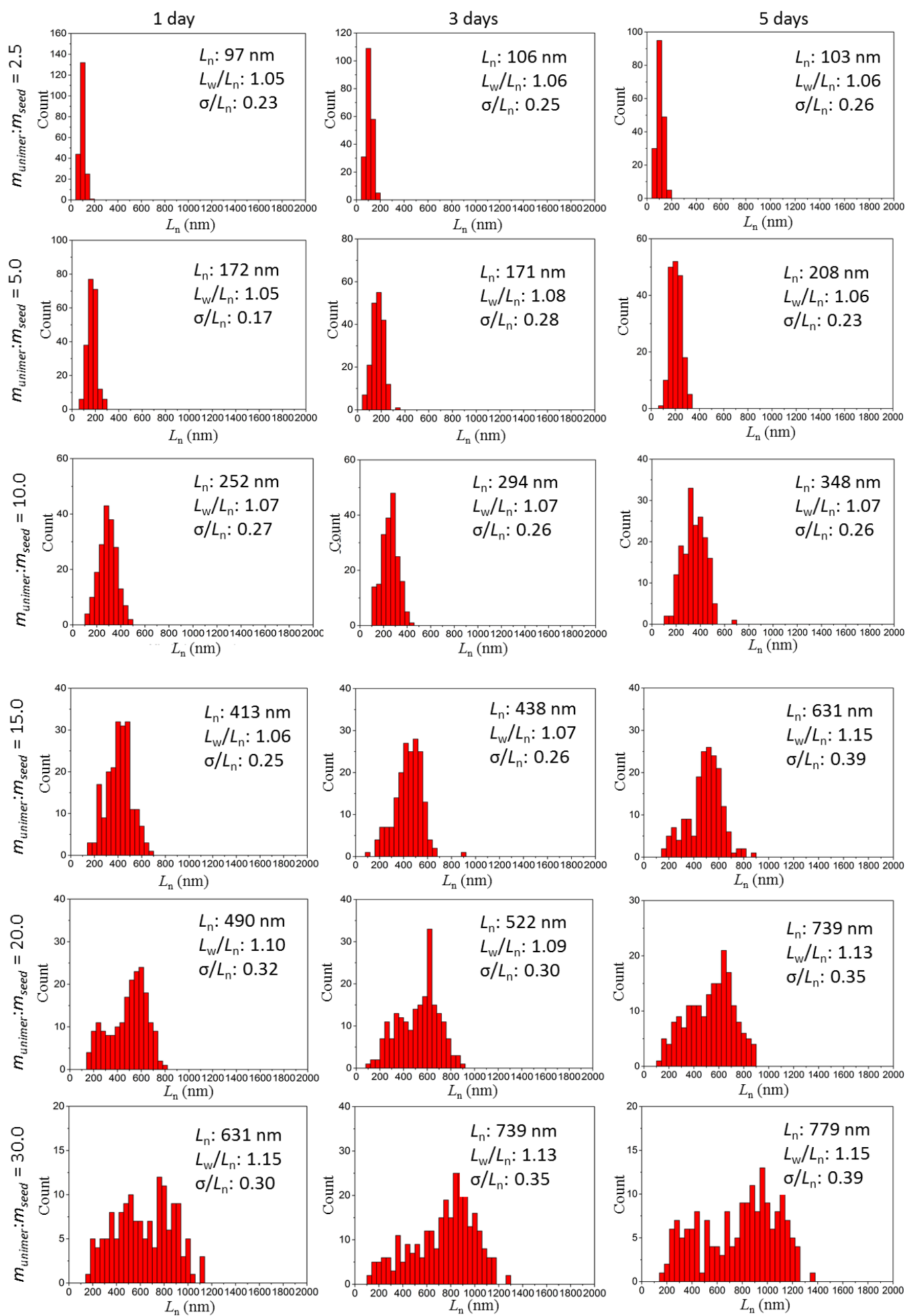

Figure S13. Contour length histograms of elongated PLLA $47-b$-PNIPAm 267 micelles in kinetic studies prepared by seeded growth off seed micelles $\left(L_{\mathrm{n}}=36 \mathrm{~nm}, L_{\mathrm{w}} / L_{\mathrm{n}}=1.10, \sigma / L_{\mathrm{n}}: 0.26\right)$ in EtOH after addition of unimers (in DMSO) with unimer-to-seed mass ratios of 2.5, 5.0, 10.0, 15.0, 20.0 and 30.0. 

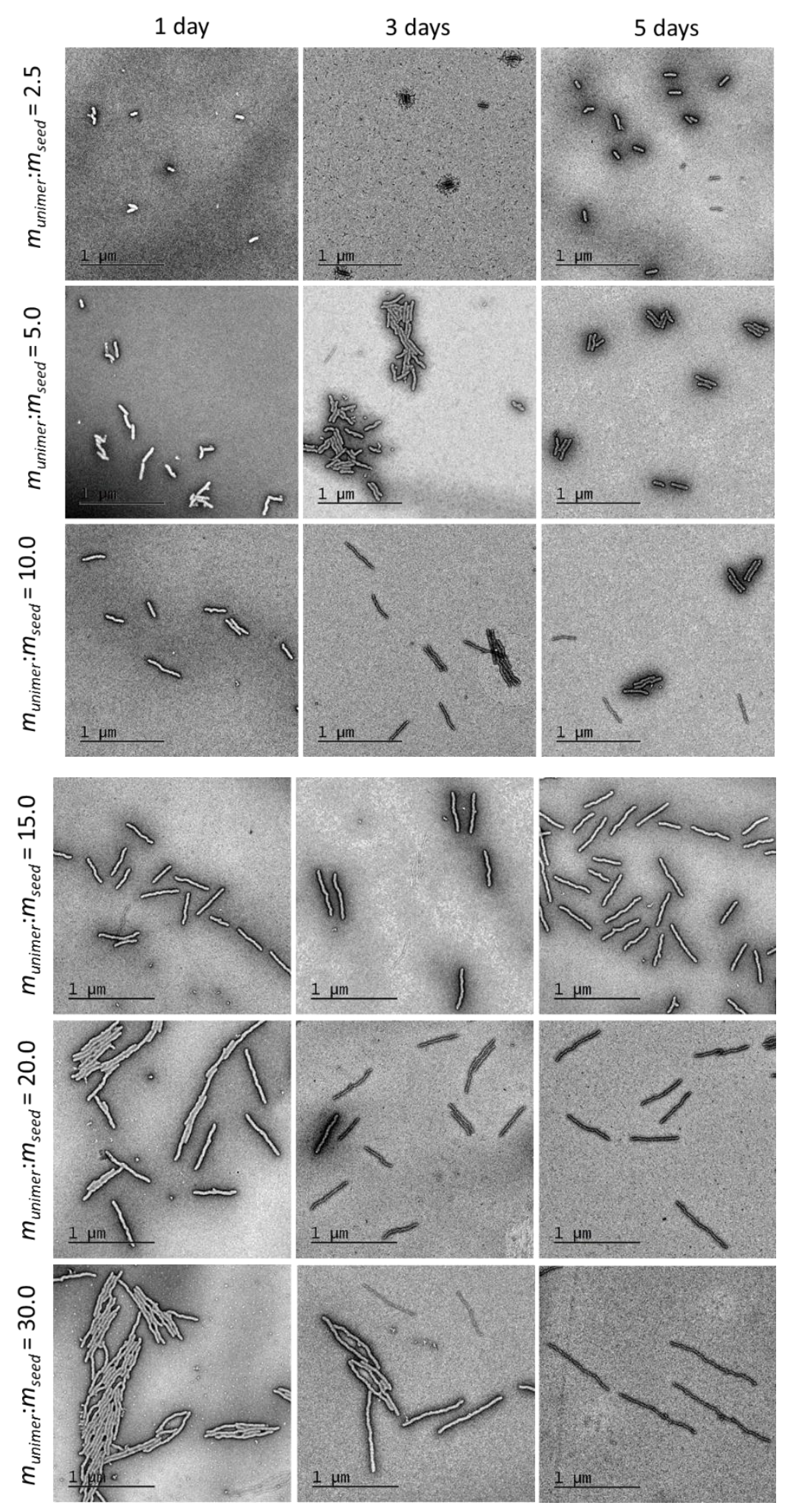

Figure S14. TEM images of uniform PLLA $47-b$-PNIPAm 267 micelles in kinetic studies prepared by seeded growth off seed micelles $\left(L_{\mathrm{n}}=36 \mathrm{~nm}, L_{\mathrm{w}} / L_{\mathrm{n}}=1.10, \sigma / L_{\mathrm{n}}: 0.26\right)$ in TFE/EtOH with volume ratios of 3:97, 3:97, 5:95, 8:92, 10:90 and 15:85 after addition of unimers (in DMSO) with unimer-to-seed mass ratios of 2.5, 5.0, 10.0, 15.0, 20.0 and 30.0, respectively; TEM samples were stained with a $2 \mathrm{wt} \%$ solution of uranyl acetate in $\mathrm{EtOH}$. 

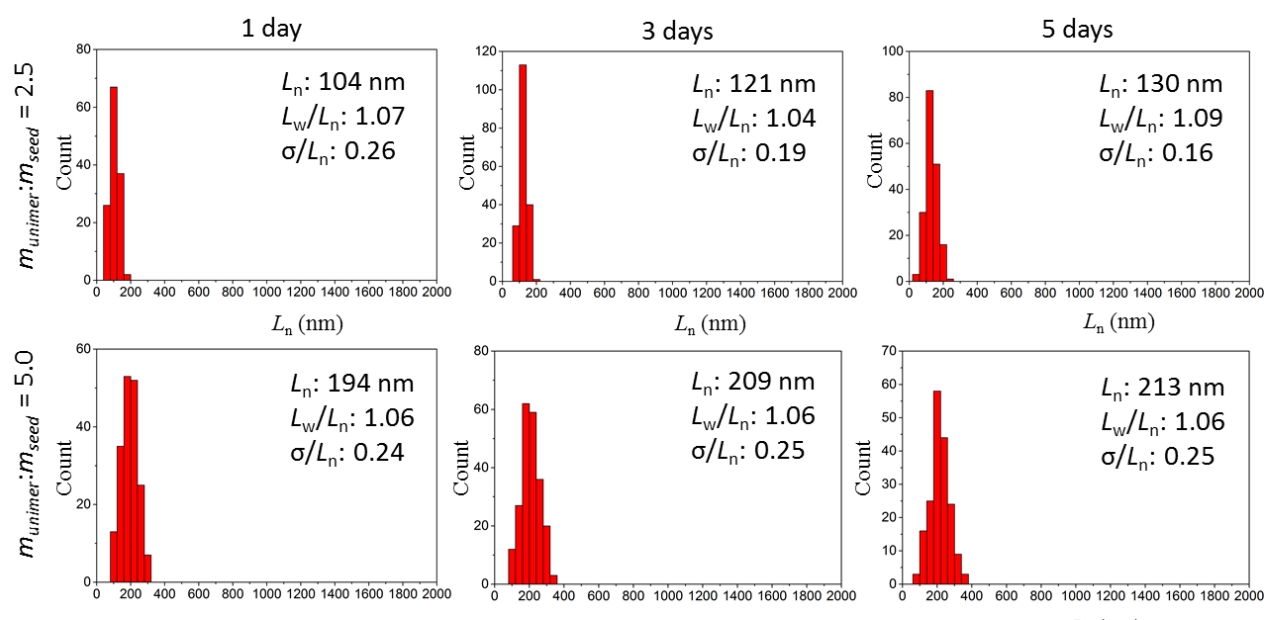

$L_{\mathrm{n}}(\mathrm{nm})$
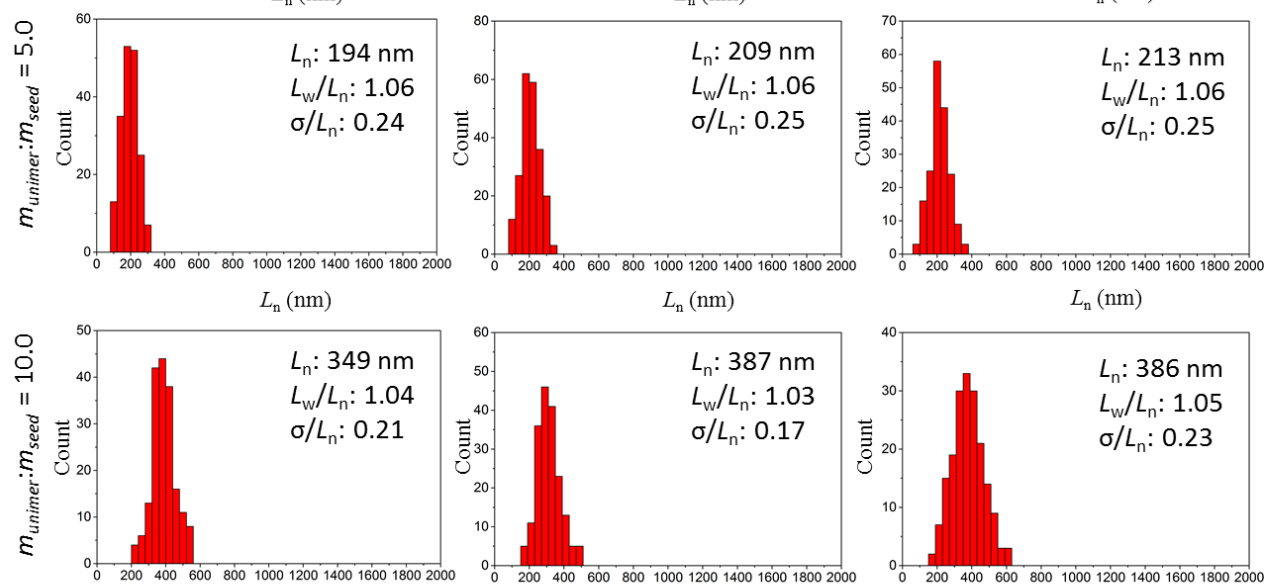

$L_{\mathrm{n}}(\mathrm{nm})$

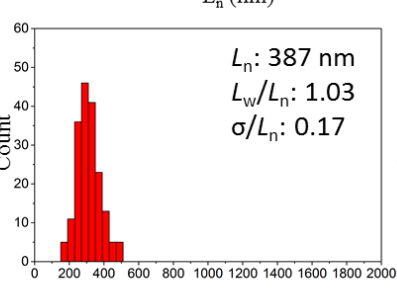

$L_{\mathrm{n}}(\mathrm{nm})$

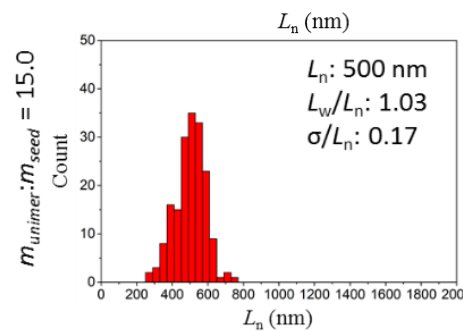

$L_{\mathrm{n}}(\mathrm{nm})$
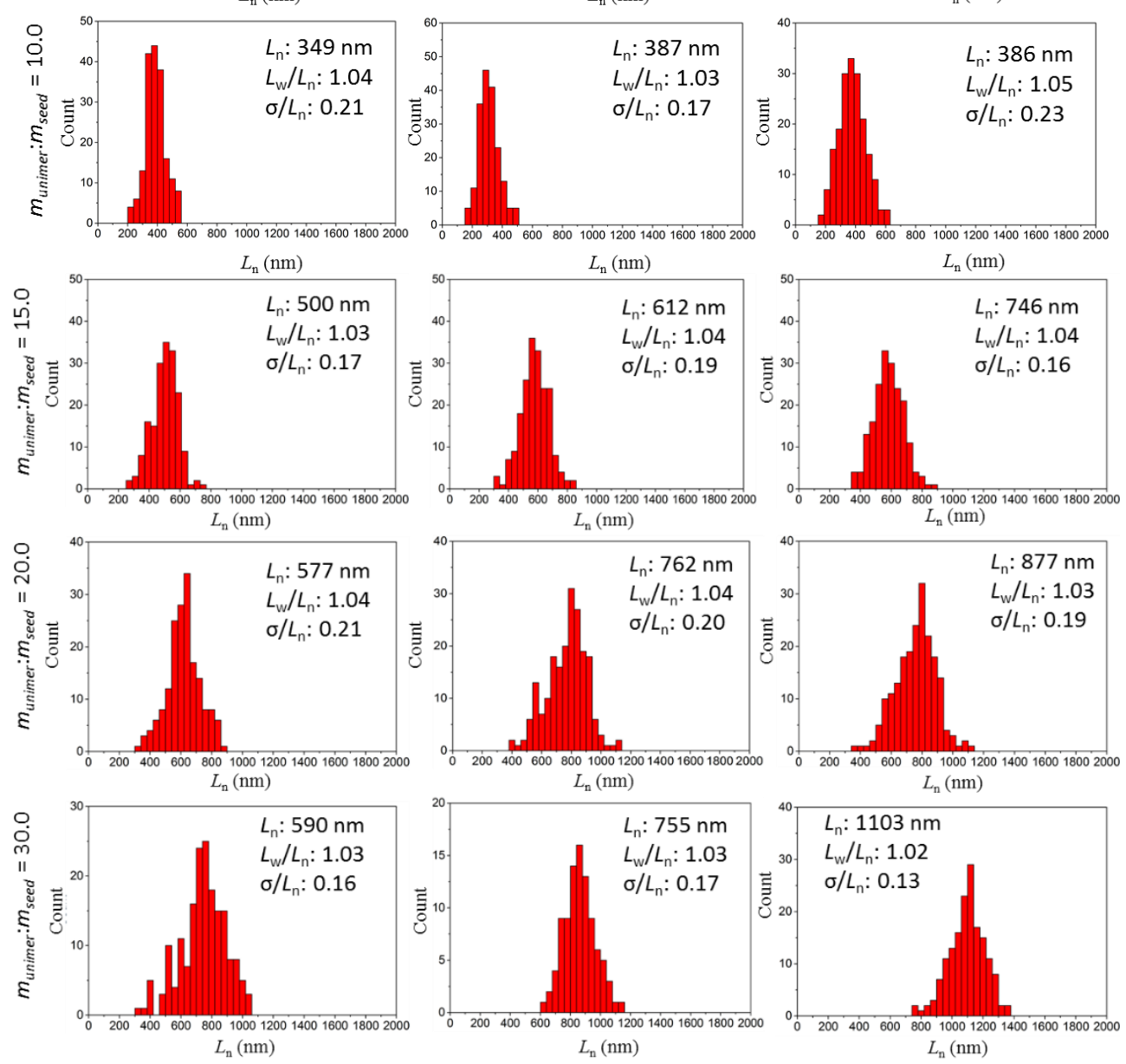

Figure S15. Contour length histograms of uniform PLLA $47-b-$ PNIPAm $_{267}$ micelles in kinetic studies prepared by seeded growth off seed micelles $\left(L_{\mathrm{n}}=36 \mathrm{~nm}, L_{\mathrm{w}} / L_{\mathrm{n}}=1.10, \sigma / L_{\mathrm{n}}: 0.26\right)$ in TFE/EtOH with volume ratios of 3:97, 3:97, 5:95, 8:92, 10:90 and 15:85 after addition of unimers (in DMSO) with unimer-to-seed mass ratios of $2.5,5.0,10.0,15.0,20.0$ and 30.0, respectively. 

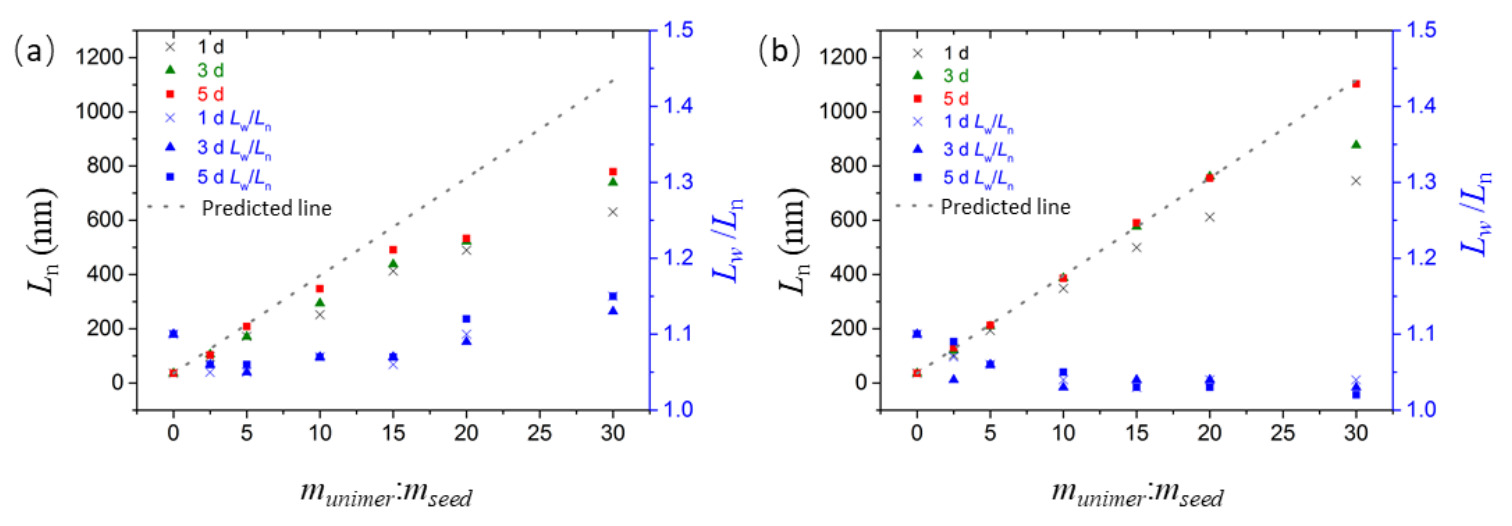

Figure S16. Plots of micelle lengths as a function of time monitored over 5 days. Unimers (in DMSO) was added to seed solutions $\left(0.5 \mathrm{mg} / \mathrm{mL}, L_{\mathrm{n}}=36 \mathrm{~nm}\right.$ ) in (a) EtOH and in (b) TFE/EtOH ( $v: v=3: 97$, 3:97, 5:95, 8:92, 10:90 and 15:85 for $m_{\text {unimer }}: m_{\text {seed }}=2.5,5.0,10.0,15.0,20.0$ and 30.0, respectively). Black label - micelle average length; blue label $-L_{\mathrm{w}} / L_{\mathrm{n}}$.

Table S2. Data summary of kinetic studies on seeded-growth of PLLA $47-b$-PNIPAm 267 micelles in EtOH over 5 days.

\begin{tabular}{|c|c|c|c|c|c|c|c|c|}
\hline \multirow{2}{*}{\multicolumn{2}{|c|}{ In EtOH }} & \multicolumn{7}{|c|}{$m_{\text {unimer }}: m_{\text {seed }}$} \\
\hline & & $\begin{array}{c}0 \\
\text { (seed) }\end{array}$ & 2.5 & 5.0 & 10.0 & 15.0 & 20.0 & 30.0 \\
\hline \multirow{6}{*}{$1 \mathrm{~d}$} & $L_{\mathrm{n}}$ & 36 & 97 & 172 & 252 & 413 & 490 & 631 \\
\hline & $L_{w}$ & 40 & 102 & 180 & 271 & 439 & 540 & 728 \\
\hline & $L_{\mathrm{w}} / L_{\mathrm{n}}$ & 1.1 & 1.05 & 1.05 & 1.07 & 1.06 & 1.10 & 1.15 \\
\hline & $\sigma$ & 9.4 & 22.5 & 36.7 & 68.2 & 104.7 & 157.2 & 246.6 \\
\hline & $L_{n} /$ eq & - & 27 & 29 & 23 & 26 & 23 & 20 \\
\hline & $\sigma / L_{n}$ & 0.26 & 0.23 & 0.17 & 0.27 & 0.25 & 0.32 & 0.39 \\
\hline \multirow{6}{*}{$3 d$} & $L_{n}$ & 36 & 106 & 171 & 294 & 438 & 522 & 739 \\
\hline & $L_{\mathrm{w}}$ & 40 & 113 & 184 & 300 & 468 & 570 & 830 \\
\hline & $L_{\mathrm{w}} / L_{\mathrm{n}}$ & 1.1 & 1.06 & 1.08 & 1.07 & 1.07 & 1.09 & 1.13 \\
\hline & $\sigma$ & 9.4 & 26.7 & 48.2 & 75.9 & 113.8 & 155.8 & 258.5 \\
\hline & $L_{n} /$ eq & - & 30 & 28 & 27 & 27 & 25 & 24 \\
\hline & $\sigma / L_{n}$ & 0.26 & 0.25 & 0.28 & 0.26 & 0.26 & 0.30 & 0.35 \\
\hline \multirow{6}{*}{$5 d$} & $L_{n}$ & 36 & 103 & 208 & 348 & 491 & 533 & 779 \\
\hline & $L_{w}$ & 40 & 109 & 221 & 373 & 527 & 596 & 899 \\
\hline & $L_{\mathrm{w}} / L_{\mathrm{n}}$ & 1.1 & 1.06 & 1.06 & 1.07 & 1.07 & 1.12 & 1.15 \\
\hline & $\sigma$ & 9.4 & 26.6 & 47.9 & 92.2 & 133.9 & 183.5 & 304.1 \\
\hline & $L_{n} /$ eq & - & 29 & 35 & 32 & 31 & 25 & 26 \\
\hline & $\sigma / L_{n}$ & 0.26 & 0.26 & 0.23 & 0.26 & 0.27 & 0.34 & 0.39 \\
\hline
\end{tabular}


Table S3. Data summary of kinetic studies over 5 days on seeded-growth of PLLA ${ }_{47}-b$-PNIPAm 267 micelles in TFE/EtOH with volume ratios of 3:97, 3:97, 5:95, 8:92, 10:90 and 15:85 after the addition of unimers (in DMSO) with unimer-to-seed mass ratios of 2.5, 5.0, 10.0, 15.0, 20.0 and 30.0, respectively.

\begin{tabular}{|c|c|c|c|c|c|c|c|c|}
\hline \multirow{2}{*}{\multicolumn{2}{|c|}{ In TFE/EtOH }} & \multicolumn{7}{|c|}{$m_{\text {unimer }}: m_{\text {seed }}$} \\
\hline & & $\begin{array}{c}0 \\
\text { (seed) }\end{array}$ & 2.5 & 5.0 & 10.0 & 15.0 & 20.0 & 30.0 \\
\hline \multirow{6}{*}{$1 d$} & $L_{\mathrm{n}}$ & 36 & 104 & 194 & 349 & 500 & 612 & 746 \\
\hline & $L_{\mathrm{w}}$ & 40 & 111 & 205 & 365 & 515 & 636 & 778 \\
\hline & $L_{\mathrm{w}} / L_{\mathrm{n}}$ & 1.1 & 1.07 & 1.06 & 1.04 & 1.03 & 1.04 & 1.04 \\
\hline & $\sigma$ & 9.4 & 27.3 & 47.5 & 73.6 & 85.1 & 120.4 & 155.3 \\
\hline & $L_{\mathrm{n}} / \mathrm{eq}$ & - & 30 & 32 & 31 & 31 & 29 & 24 \\
\hline & $\sigma / L_{n}$ & 0.26 & 0.26 & 0.24 & 0.21 & 0.17 & 0.19 & 0.16 \\
\hline \multirow{6}{*}{$3 d$} & $L_{n}$ & 36 & 121 & 209 & 387 & 577 & 762 & 877 \\
\hline & $L_{w}$ & 40 & 125 & 222 & 399 & 600 & 795 & 908 \\
\hline & $L_{\mathrm{w}} / L_{\mathrm{n}}$ & 1.1 & 1.04 & 1.06 & 1.03 & 1.04 & 1.04 & 1.03 \\
\hline & $\sigma$ & 9.4 & 22.5 & 51.8 & 67.6 & 122.7 & 157.9 & 165.1 \\
\hline & $L_{\mathrm{n}} / \mathrm{eq}$ & - & 34 & 34 & 35 & 36 & 36 & 28 \\
\hline & $\sigma / L_{n}$ & 0.26 & 0.19 & 0.25 & 0.17 & 0.21 & 0.20 & 0.19 \\
\hline \multirow{6}{*}{$5 d$} & $L_{n}$ & 36 & 130 & 213 & 386 & 590 & 755 & 1103 \\
\hline & $L_{\mathrm{w}}$ & 40 & 142 & 228 & 406 & 606 & 779 & 1122 \\
\hline & $L_{\mathrm{w}} / L_{\mathrm{n}}$ & 1.1 & 1.09 & 1.06 & 1.05 & 1.03 & 1.03 & 1.02 \\
\hline & $\sigma$ & 9.4 & 20.4 & 55.1 & 90.1 & 95.7 & 133.6 & 138.2 \\
\hline & $L_{n} /$ eq & - & 37 & 36 & 35 & 37 & 36 & 35 \\
\hline & $\sigma / L_{n}$ & 0.26 & 0.16 & 0.25 & 0.23 & 0.16 & 0.17 & 0.13 \\
\hline
\end{tabular}



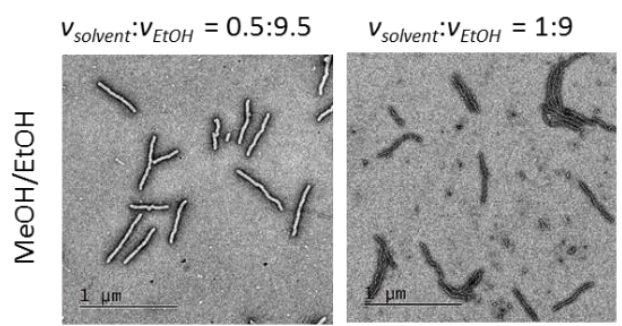

$v_{\text {solvent }}: v_{E t O H}=1.5: 8.5$
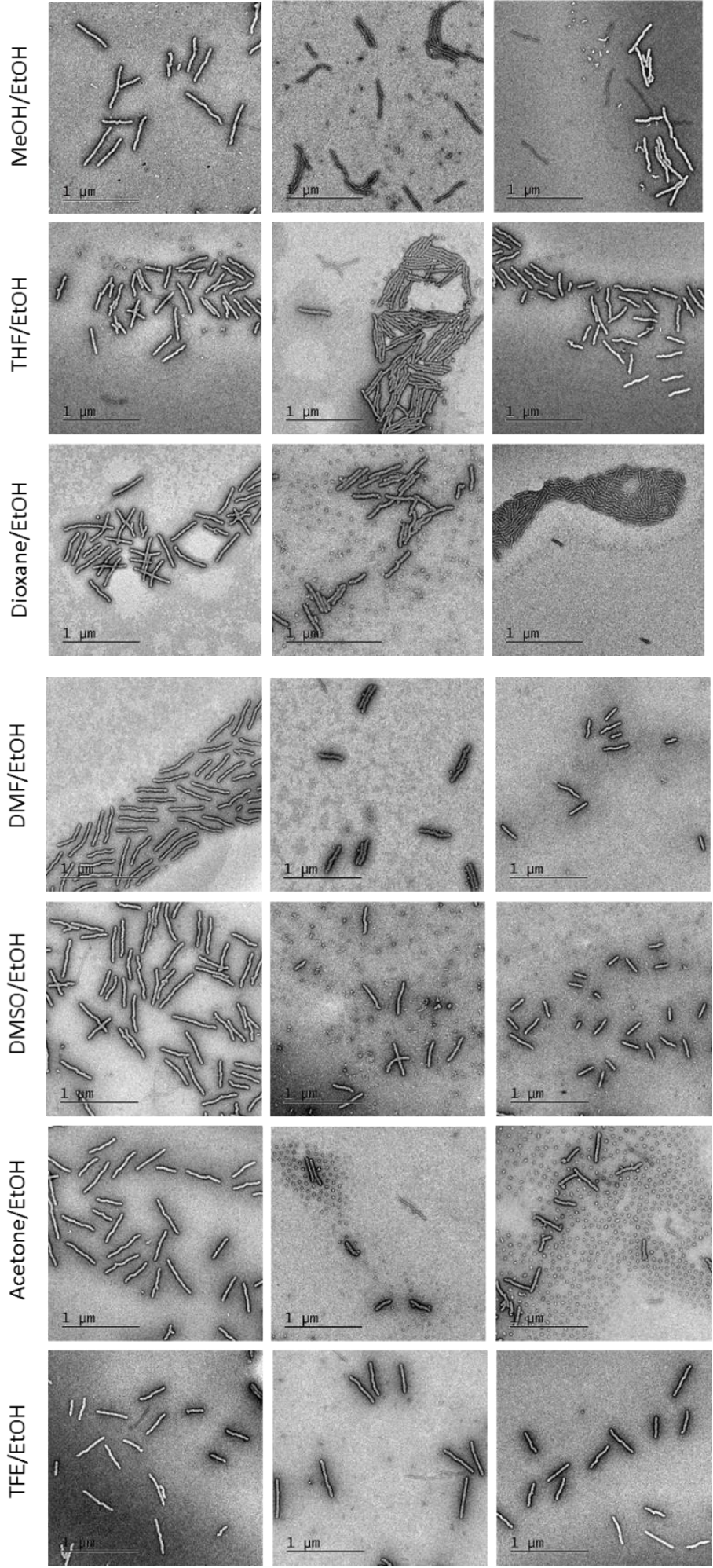

Figure S17. TEM images of uniform PLLA PL- $_{47}-$ PNIPAm $_{267}$ micelles in solvent effect studies prepared by adding unimers $\left(m_{\text {unimer }}: m_{\text {seed }}=15.0\right.$, in DMSO) to seed micelles $\left(L_{\mathrm{n}}=36 \mathrm{~nm}, L_{\mathrm{w}} / L_{\mathrm{n}}=1.10, \sigma / L_{\mathrm{n}}\right.$ : $0.26)$ in TFE/EtOH ( $v: v=0.5: 9.5,1.0: 9.0$ and 1.5:8.5); TEM samples were stained with a $2 \mathrm{wt} \%$ solution of uranyl acetate in EtOH. Inset: $100 \mathrm{~nm}$. 

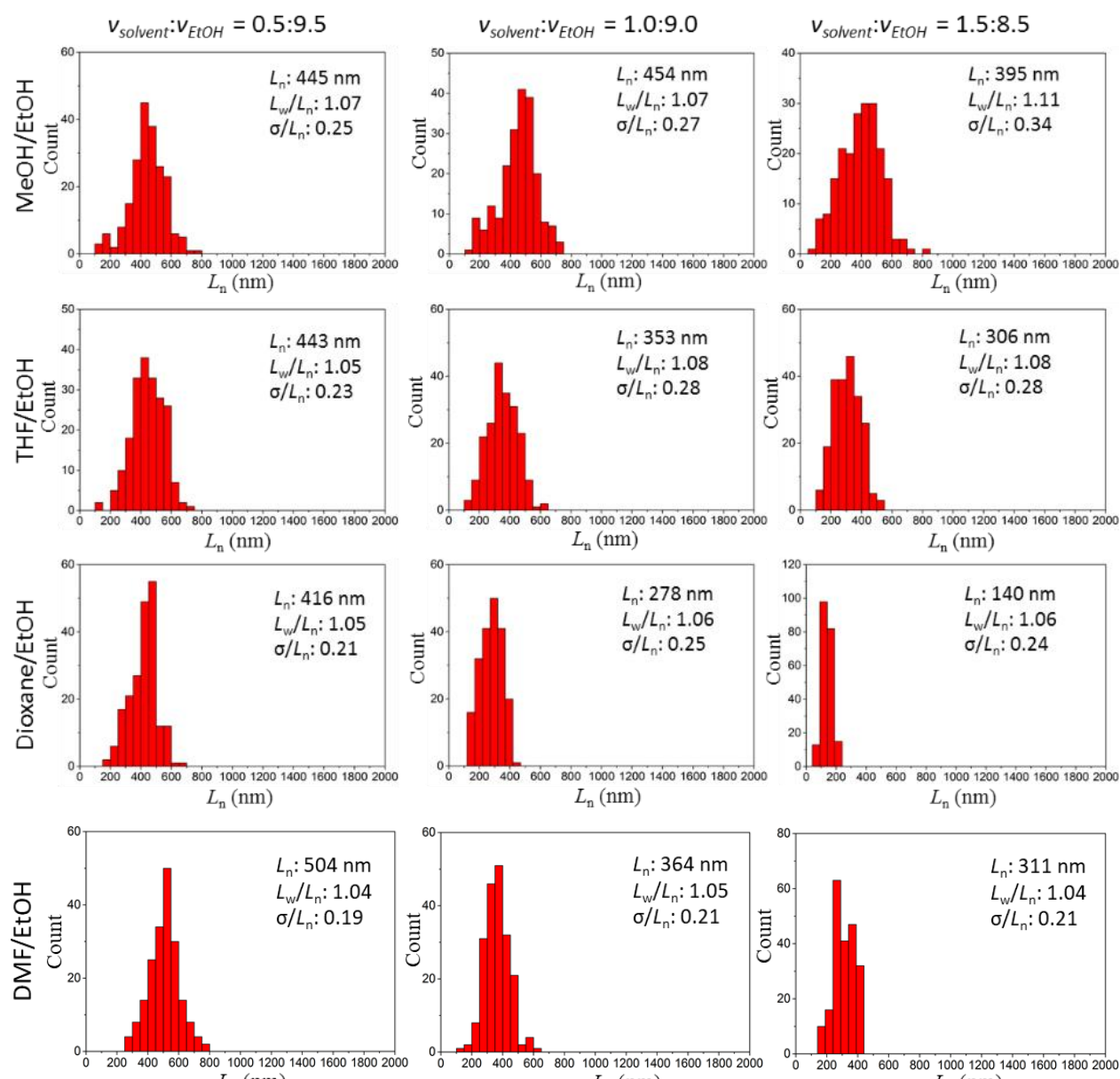

$L_{\mathrm{n}}(\mathrm{nm})$
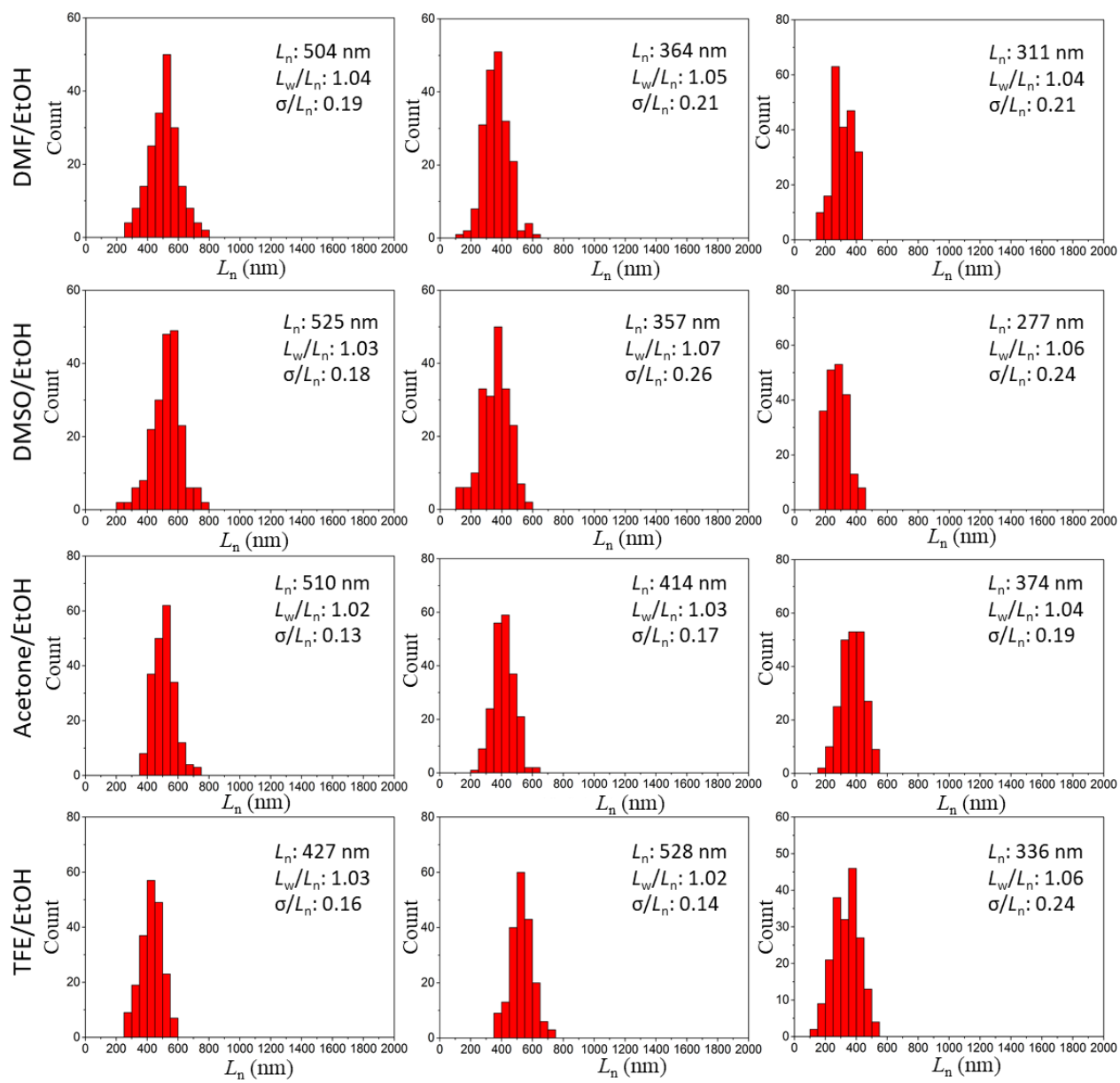

Figure S18. Contour length histograms of uniform PLLA $47-b$-PNIPAm 267 micelles in solvent effect studies prepared by adding unimers ( $m_{\text {unimer }}: m_{\text {seed }}=15.0$, in DMSO) to seed micelles $\left(L_{\mathrm{n}}=36 \mathrm{~nm}, L_{\mathrm{w}} / L_{\mathrm{n}}\right.$ $\left.=1.10, \sigma / L_{\mathrm{n}}: 0.26\right)$ in TFE/EtOH $(v: v=0.5: 9.5,1.0: 9.0$ and 1.5:8.5). 


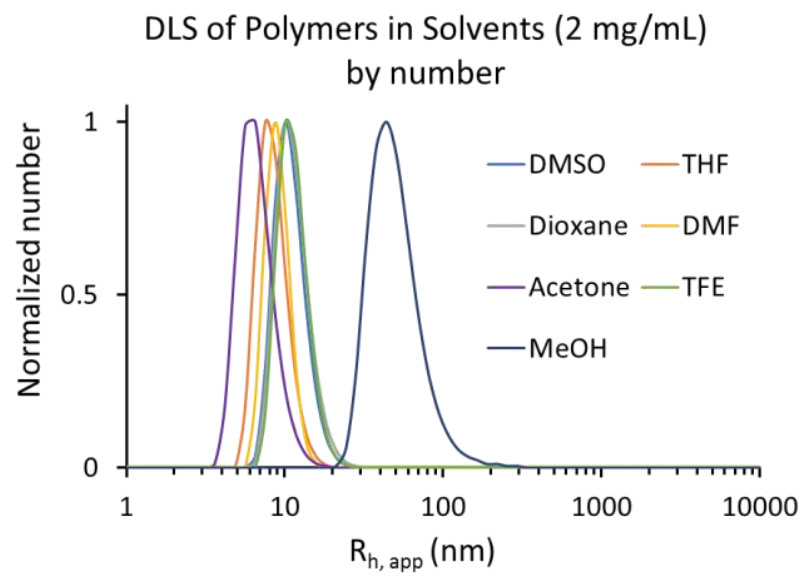

Figure S19. Solutions of PLLA $47-b$-PNIPAm 267 in various solvents $(2 \mathrm{mg} / \mathrm{mL})$ characterized by DLS.

(a)

\begin{tabular}{cc}
\hline & $\log _{\text {oct }} / \mathrm{SA}$ \\
\hline PLLA & 0.002 \\
PNIPAm & -0.068 \\
EtOH & -0.0002 \\
\hline
\end{tabular}

(b)

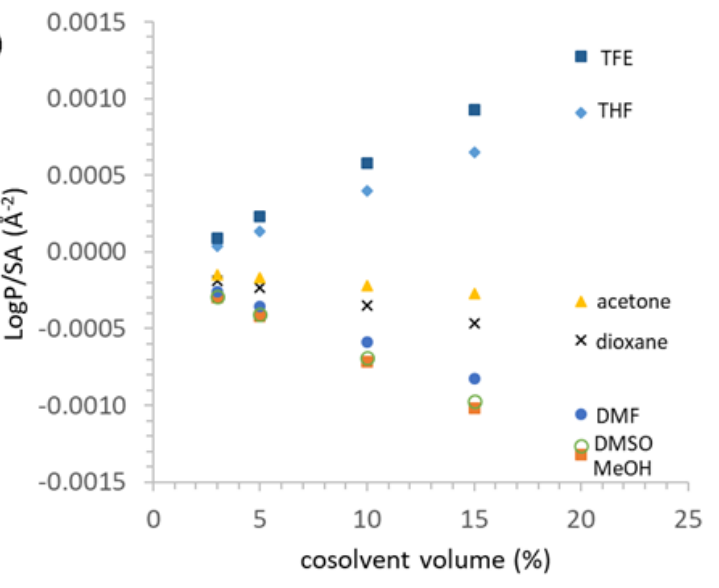

Figure S20. Calculated $\log \mathrm{P}_{\text {oct }} / \mathrm{SA}$ values of (a) PLLA, PNIPAm, EtOH and (b) solvent mixture.

Table S4. Table of Dimroth and Reichardt's Transition Energy $\left(\mathrm{E}_{\mathrm{T}}(30)\right)$ of Cosolvents.

\begin{tabular}{ccccc}
\hline Solvents & $\mathrm{E}_{\mathrm{T}}(\mathbf{3 0})$ & $\boldsymbol{\pi}^{*}$ & $\boldsymbol{\alpha}$ & $\boldsymbol{\beta}$ \\
\hline $\mathrm{H}_{2} \mathrm{O}^{*}$ & 62.3 & 1.09 & 1.17 & 0.47 \\
TFE & 61.2 & 0.73 & 1.49 & 0.00 \\
DMSO & 44.8 & 1 & 0.00 & 0.76 \\
DMF & 43.1 & 0.88 & 0.00 & 0.69 \\
Acetone & 41.6 & 0.71 & 0.08 & 0.48 \\
THF & 38.9 & 0.58 & 0.00 & 0.55 \\
1,4-Dioxane & 38.1 & 0.55 & 0.00 & 0.37 \\
MeOH & 53.6 & 0.60 & 0.98 & 0.66 \\
$n$-Pentane* & 30.2 & 0.00 & 0.00 & 0.00 \\
\hline
\end{tabular}

$\alpha$ : scale of hydrogen bonding donor acidity

$\beta$ : scale of hydrogen bonding acceptor basicity

$\pi^{*}$ : solvatochromic parameter

$\mathrm{E}_{\mathrm{T}}$ : Dimroth and Reichardt's Transition Energy, a re-

examined data from $E_{T}(30)_{0}$

$\mathrm{E}_{\mathrm{T}}(30)=E_{T}(30)_{0}+s(\pi *+d \delta)+\mathrm{a} \alpha+\mathrm{b} \beta$

* Scale reference of $\mathrm{E}_{\mathrm{T}}$ 
(a)

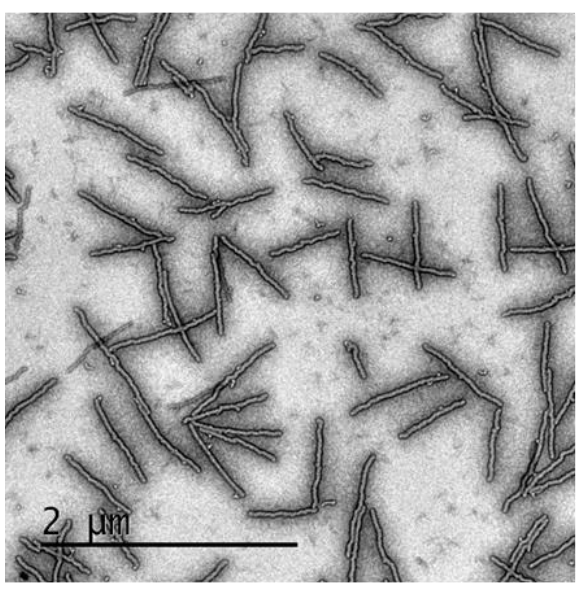

(b)

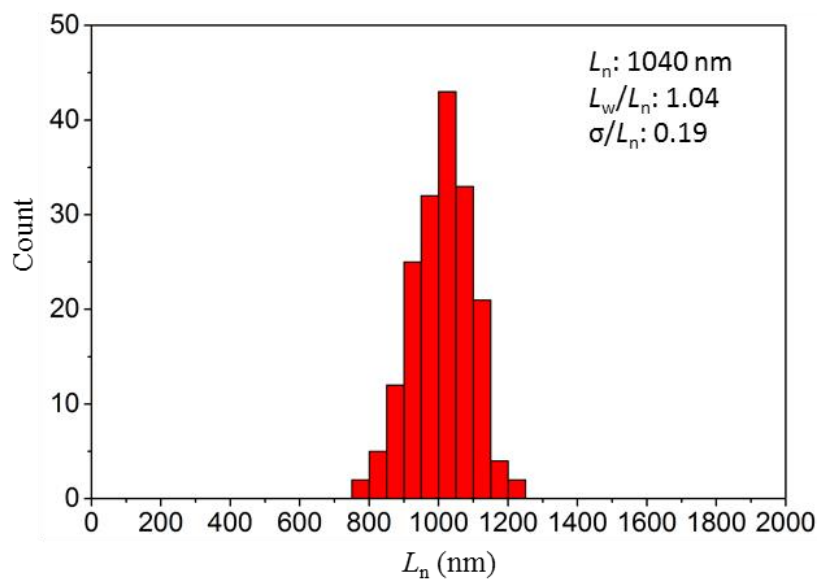

Figure S21. (a) TEM images of PLLA $_{47}-b$-PNIPAm 267 micelles prepared for characterization experiments with length of $L_{\mathrm{n}}=1040 \mathrm{~nm}\left(L_{\mathrm{w}} / L_{\mathrm{n}}=1.04\right)$; (b) contour length histogram of measured length data. TEM image was stained with uranyl acetate solution $(2 \%$ in EtOH).

(a)

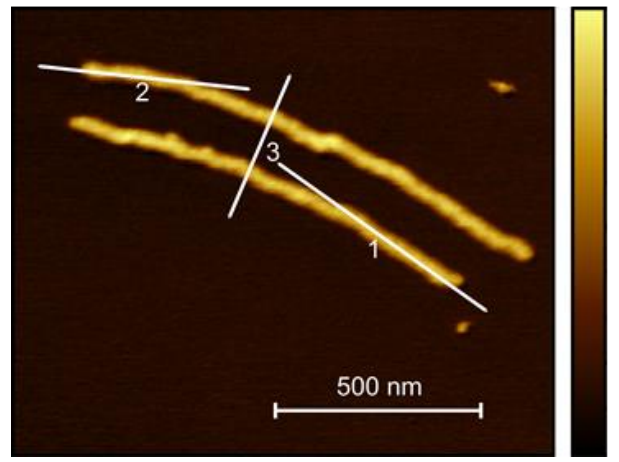

(b)

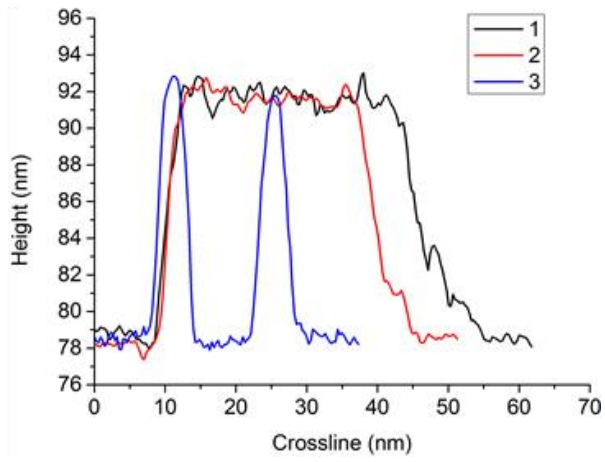

Figure S22. AFM images of PLLA $47-b$-PNIPAm 267 micelles with controlled length. (a) Height image of micelles; (b) height profile by crossline measurements.

(a)

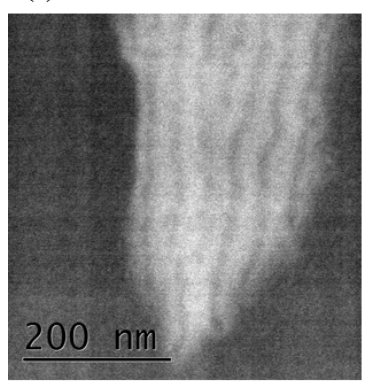

(b)

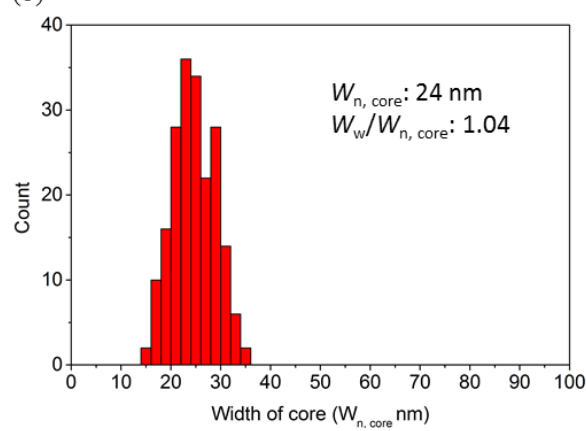

(c)

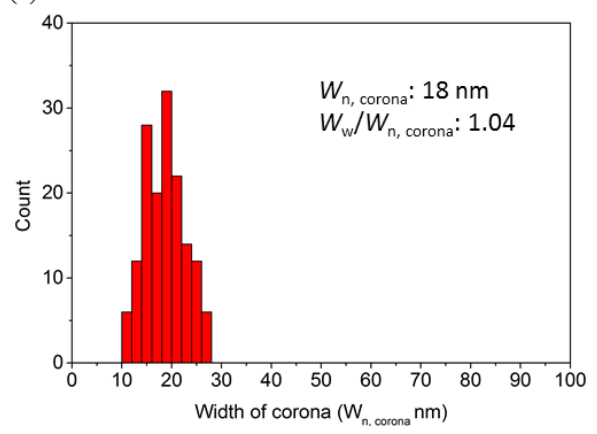

Figure S23. (a) STEM images of PLLA $47-b$-PNIPAm 267 micelles in dark-field (white area is corona while dark area is core), (b) contour width histograms of core width measurement, and (c) contour width histograms of corona width on each side of the core. 

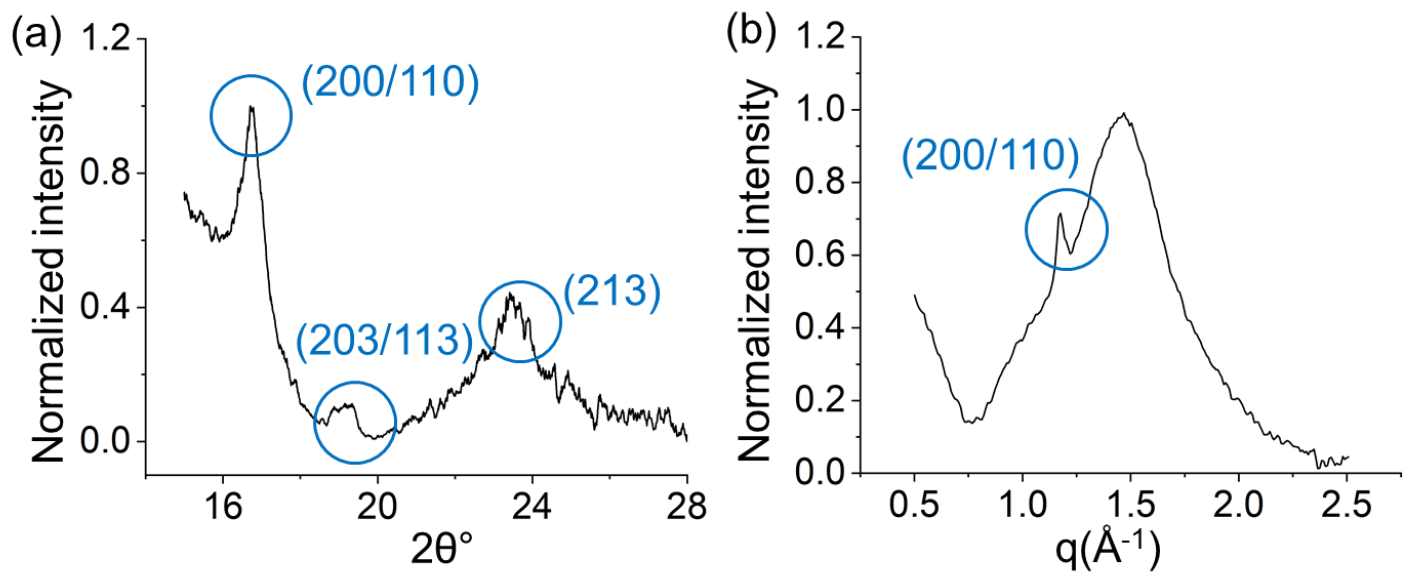

Figure S24. (a) PXRD profile of PLLA ${ }_{47}-b$-PNIPAm 267 micelles; (b) normalized intensity by WAXS for PLLA $47-b$-PNIPAm 267 micelles in EtOH with $30 \mathrm{mg} / \mathrm{mL}$. The blue circles identify the Bragg peaks.

(a)

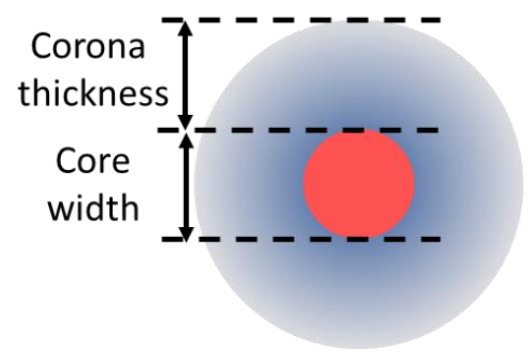

(b)

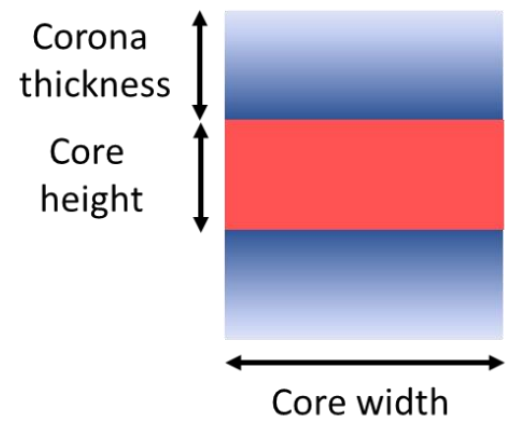

Figure S25. Schematic representation of the models used to fit SAXS data. (a) Model 1 has a homogeneous circular cross-section core (red) and a surrounding corona (blue) with decaying density; (b) Model 2 has a homogeneous rectangular cross-section core (red) with a decaying density corona (blue) attached on the long core edges.

(a)

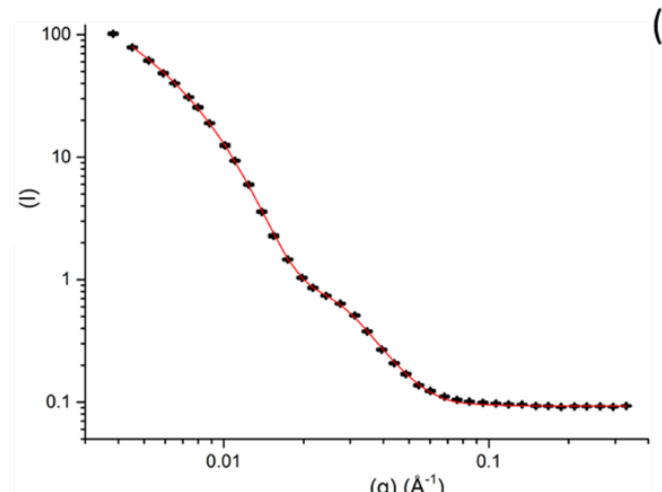

(b)

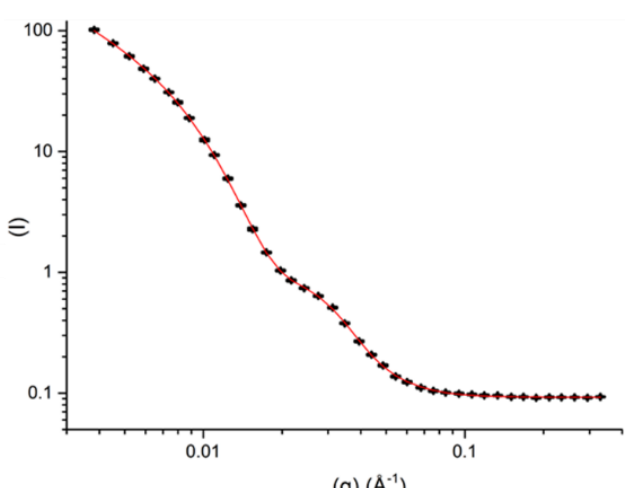

(q) $\left(\AA^{-1}\right)$

Figure S26. Plot of I vs $q$ from SAXS data of a $4 \mathrm{mg} / \mathrm{mL}$ suspension of PLLA $47-b$-PNIPAm 267 micelles (black in a and b) and fitting from Model 1 (red in a) and Model 2 (red in b) 
(a)

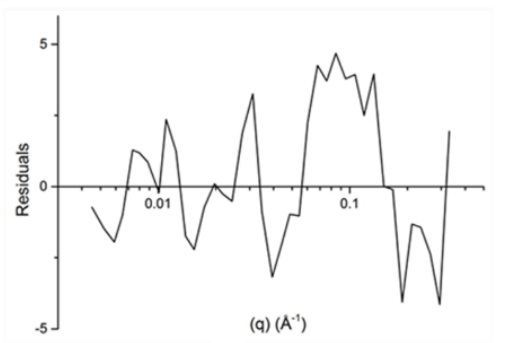

(b)

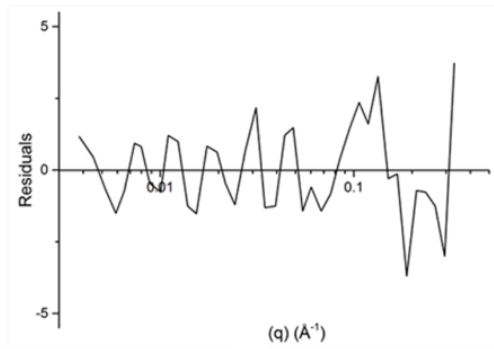

Figure S27. Plot of residual vs q from Model 1 fitting data (a) and Model 2 fitting data (b).

(a)

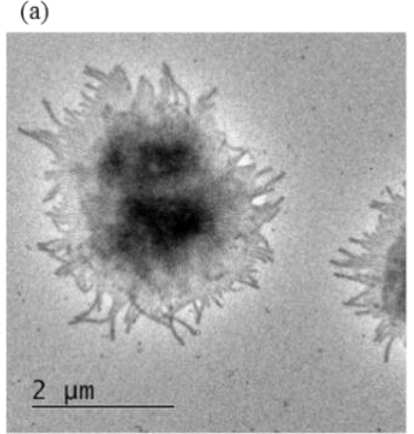

(b)

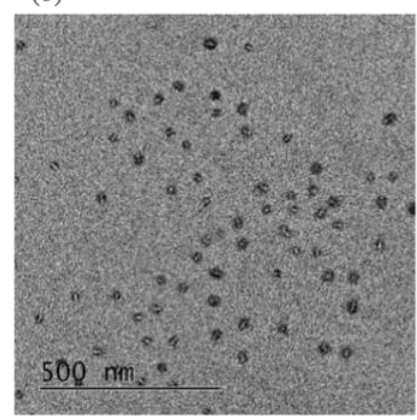

(c)

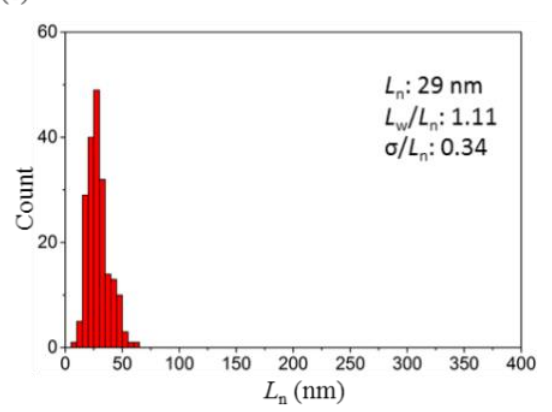

Figure S28. (a) Polydisperse PLLA $47-b-\mathrm{P}_{2} \mathrm{VP}_{503}$ micelles in DMSO/EtOH (1:9) with a concentration of $0.5 \mathrm{mg} / \mathrm{ml}$ prepared by heating the polymer in DMSO/EtOH (1:9) at $70{ }^{\circ} \mathrm{C}$ for $2 \mathrm{~h}$ followed by slow cooling over $2.5 \mathrm{~h}$; (b) seeds prepared by sonication of polydisperse micelles at $0{ }^{\circ} \mathrm{C}$ for $2 \mathrm{~h}$ in a sonic cleaning bath; (c) contour length histogram of measured seeds length, $L_{\mathrm{n}}=36 \mathrm{~nm}, L_{\mathrm{w}} / L_{\mathrm{n}}=1.10$.
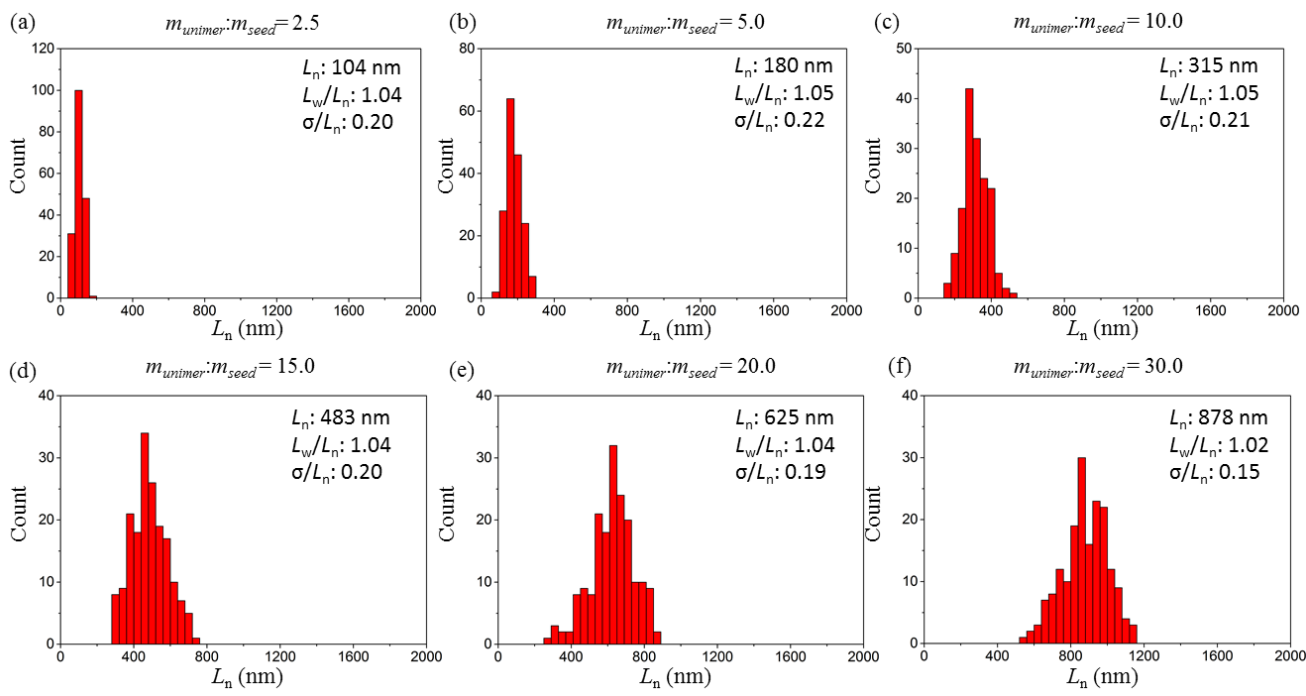

Figure S29. Contour length histograms of 5 days aged uniform $\mathrm{PLLA}_{47}-b-\mathrm{P}_{2} \mathrm{VP}_{503}$ micelles prepared by seeded growth off seed micelles $\left(L_{\mathrm{n}}=29 \mathrm{~nm}, L_{\mathrm{w}} / L_{\mathrm{n}}=1.11, \sigma / L_{\mathrm{n}}: 0.34\right)$ in TFE/EtOH with volume ratios of (a) 3:97, (b) 3:97, (c) 5:95, (d) 8:92, (e) 10:90 and (f) 15:85 after the addition of unimers (in DMSO) with unimer-to-seed mass ratios of (a) 2.5, (b) 5.0, (c) 10.0, (d) 15.0, (e) 20.0 and (f) 30.0, respectively. 
(a)

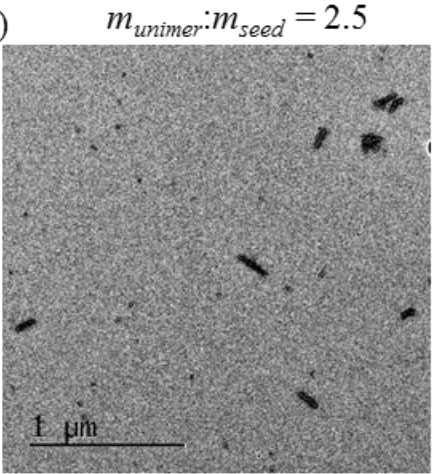

(d)

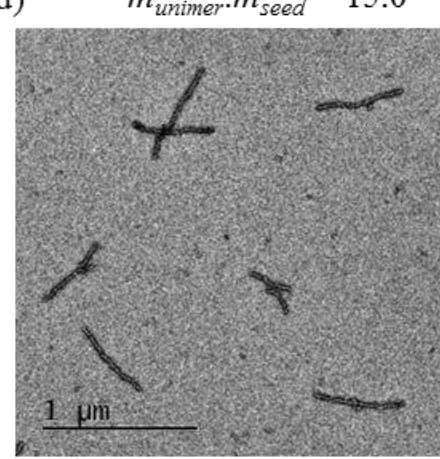

(g)

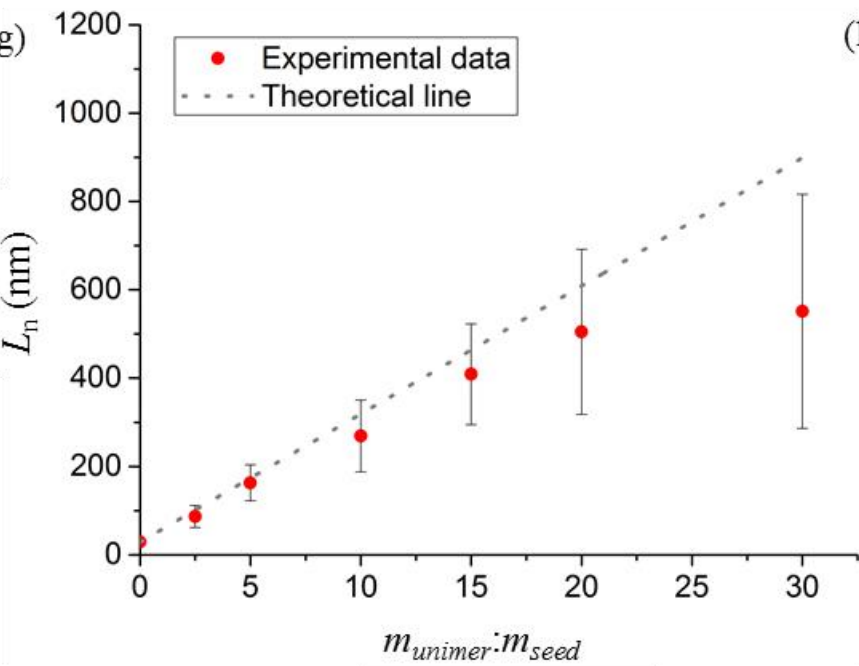

(b)

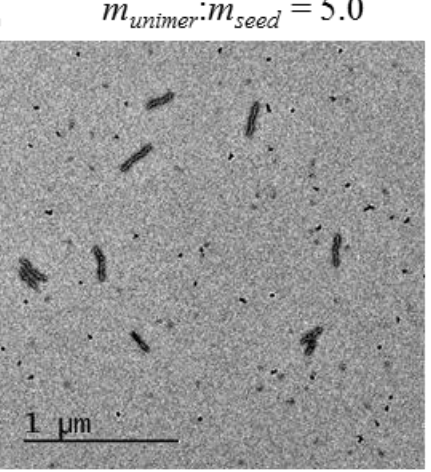

(e)

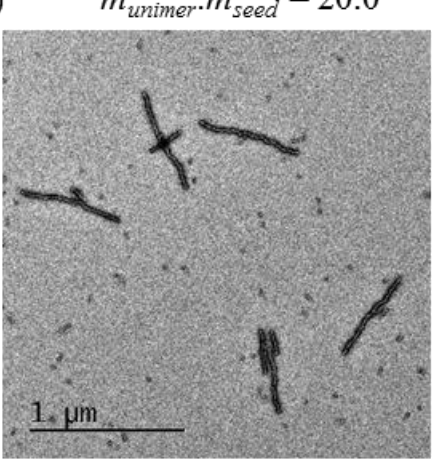

(c) $\quad m_{\text {unimer }}: m_{\text {seed }}=10.0$

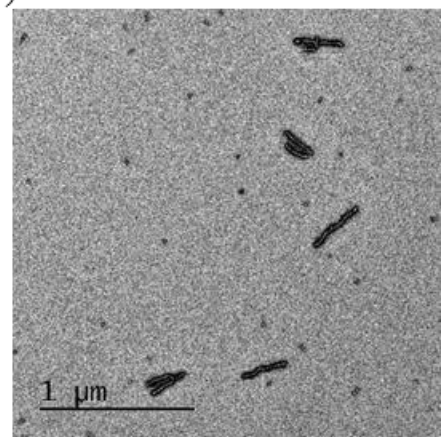

(f)

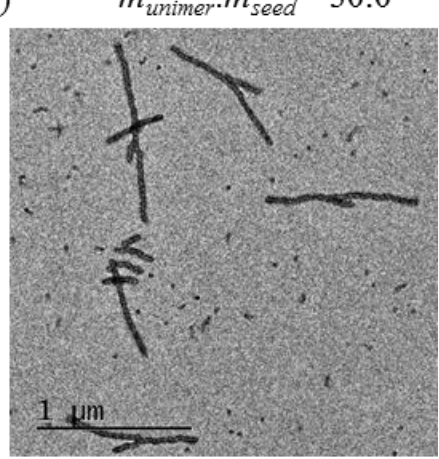

(h)

\begin{tabular}{cccc}
\hline $\begin{array}{c}m_{\text {unimer }} \\
: m_{\text {seed }}\end{array}$ & $\begin{array}{c}L_{\mathrm{n}} \\
(\mathrm{nm})\end{array}$ & $L_{\mathrm{w}} / L_{\mathrm{n}}$ & $\sigma / L_{\mathrm{n}}$ \\
\hline 2.5 & 87 & 1.08 & 0.28 \\
5.0 & 163 & 1.06 & 0.25 \\
10.0 & 269 & 1.09 & 0.30 \\
15.0 & 409 & 1.08 & 0.28 \\
20.0 & 505 & 1.14 & 0.37 \\
30.0 & 551 & 1.23 & 0.48 \\
\hline
\end{tabular}

Figure S30. TEM images of 5 days aged samples of uniform $\mathrm{PLLA}_{47}-b-\mathrm{P} 2 \mathrm{VP}_{503}$ micelles prepared by seeded growth off seed micelles $\left(L_{\mathrm{n}}=29 \mathrm{~nm}, L_{\mathrm{w}} / L_{\mathrm{n}}=1.11, \sigma / L_{\mathrm{n}}: 0.34\right)$ in EtOH after the addition of unimers (in DMSO) with unimer-to-seed mass ratios of (a) 2.5, (b) 5.0, (c) 10.0, (d) 15.0, (e) 20.0 and (f) $30.0 ;$ (g) plot of number average micelle length vs $m_{\text {unimer }}: m_{\text {seed }}$ (the error bars represent the standard deviation); (h) summary of measured length and solvent compositions; error bars were based on standard deviation. 

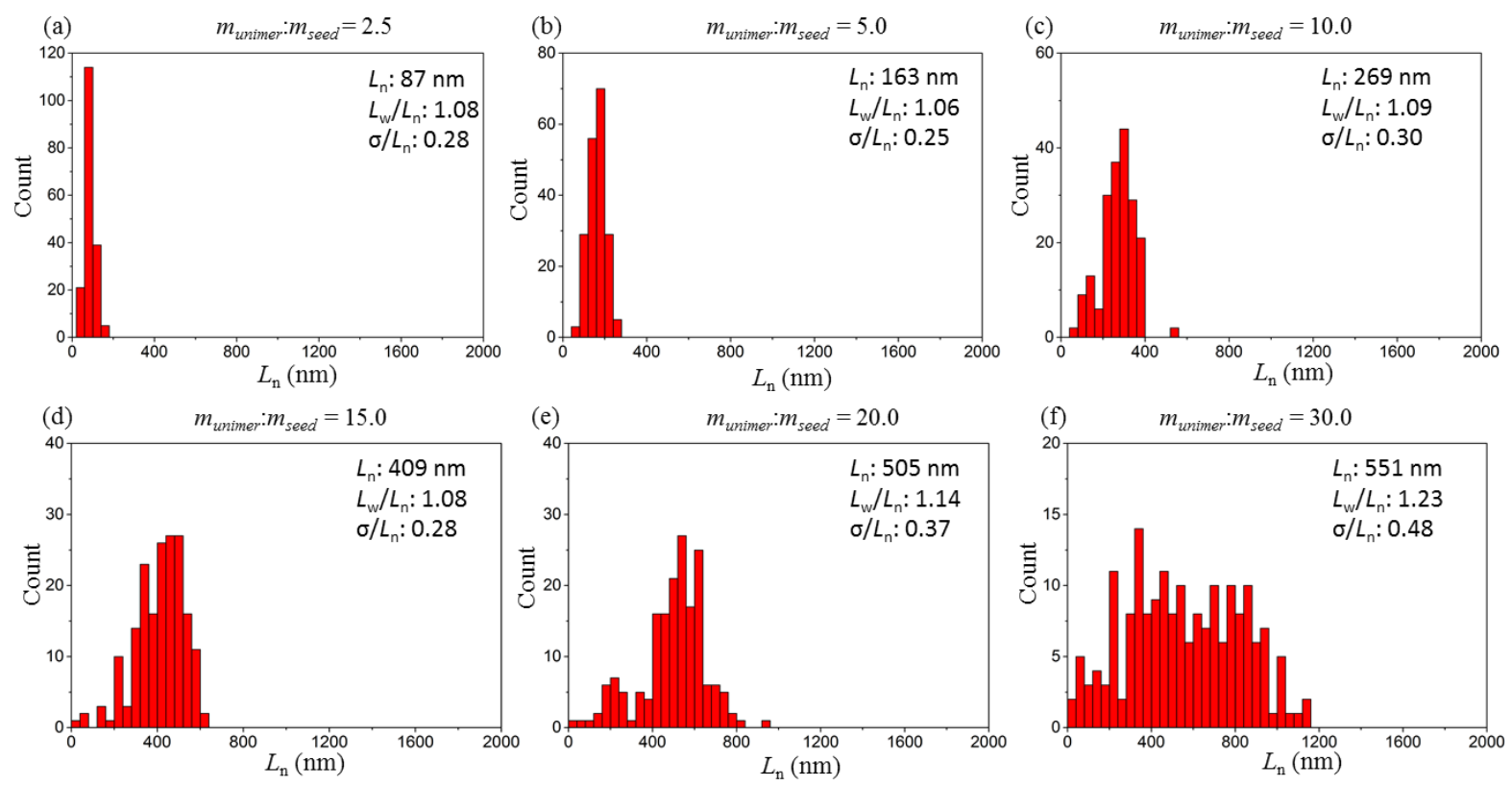

Figure S31. Contour length histograms of 5 days aged uniform PLLA $_{47}-b-\mathrm{P} 2 \mathrm{VP}_{503}$ micelles prepared by seeded growth off seed micelles $\left(L_{\mathrm{n}}=29 \mathrm{~nm}, L_{\mathrm{w}} / L_{\mathrm{n}}=1.11, \sigma / L_{\mathrm{n}}: 0.34\right)$ in EtOH after the addition of unimers (in DMSO) with unimer-to-seed mass ratios of (a) 2.5, (b) 5.0, (c) 10.0, (d) 15.0, (e) 20.0 and (f) 30.0 .

(a) Central block 1

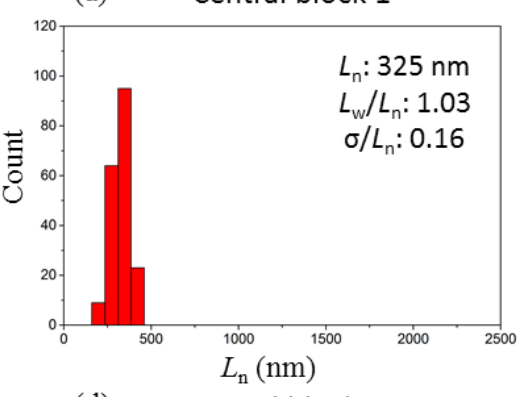

(d) Central block 2

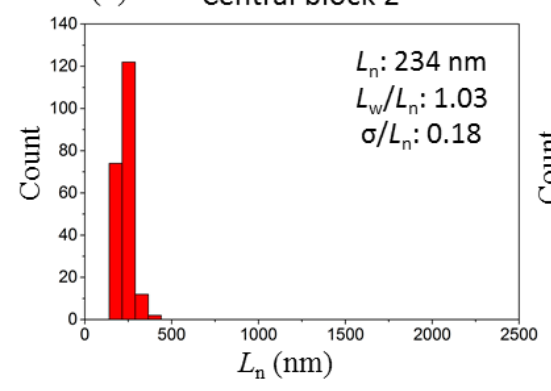

(b)

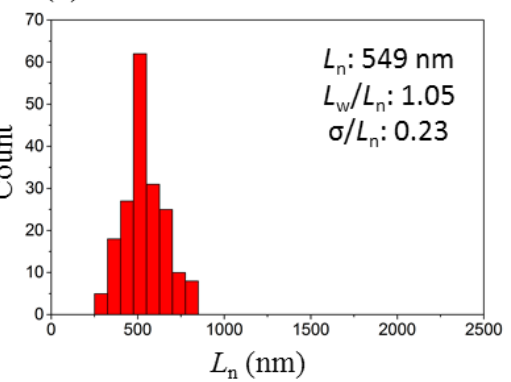

(e) Triblock 2

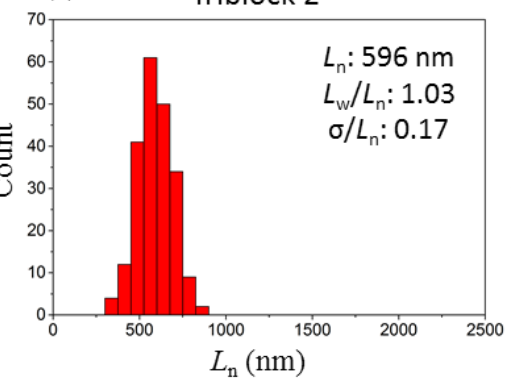

(c) Penta-block 1

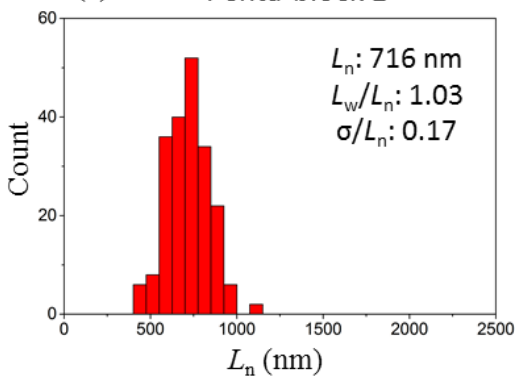

(f)

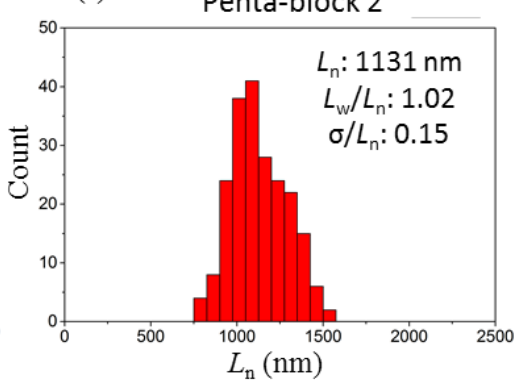

Figure S32. Contour length histograms of prepared block co-micelles: (a) central block 1, (b) triblock co-micelles 1, (c) pentablock co-micelles 1, (d) central block 2, (e) triblock co-micelles 2 and (f) pentablock co-micelles 2 . 


\section{Formula for SAXS Data Fitting with Model 2}

Model 2 (Figure S33)describes a long rigid micelle with a rectangular cross-section ( $2 a$ by $2 b$ ) core covered by corona on two faces perpendicular to the $a$ direction. The core has a uniform scattering length density, $\rho_{\text {core. }}$ The coronas have a rectangular cross-section ( $2 b$ by $c$ ) with a linear decaying scattering intensity from the inner, $\rho_{\text {in }}$, to outer side, $\rho_{\text {out. }}$ For the fitting process, the long rods approximation is used by considering the micelle length $(2 l)$ is significantly greater than the reciprocal of the minimum $q$. The scattering intensity $(\mathrm{I}(q))$ from long rods solution can be described as:

$$
I(q)=\frac{2 \pi l}{q}\left\langle F_{a}(Q \sin \phi) F_{b}(q \cos \phi)\right\rangle_{\phi}
$$

where the angle brackets represent an average over the azimuthal angle of $q$ with respect to the long micelle axis. The structure factors for the cross-section are defined as follows:

$$
\begin{gathered}
F_{b}(q)=2 b \operatorname{sinc}(q b) \\
F_{a}(q)=2 \sum_{j=1, N}\left(\rho_{j}+\rho_{j+1}\right) d_{j} \operatorname{sinc}\left(q d_{j}\right)
\end{gathered}
$$

where $\rho_{j}=\rho_{\text {core }}$ for $j=1, \rho_{j}=\rho_{\text {solvent }}$ for $j=1+N$, and $\rho_{j}=\rho_{\text {in }}+\left(\rho_{\text {out }}-\rho_{\text {in }}\right)[(\mathrm{j}-1.5) /(N-1)]$ otherwise, while $d_{j}=a$ for $j=1$ and $d_{j}=a+c(j-1) /(N-1)$ otherwise. The three dimensions, $a, b$, and $c$ were assumed to have Schultz distributions and the expression was averaged numerically over these distributions.

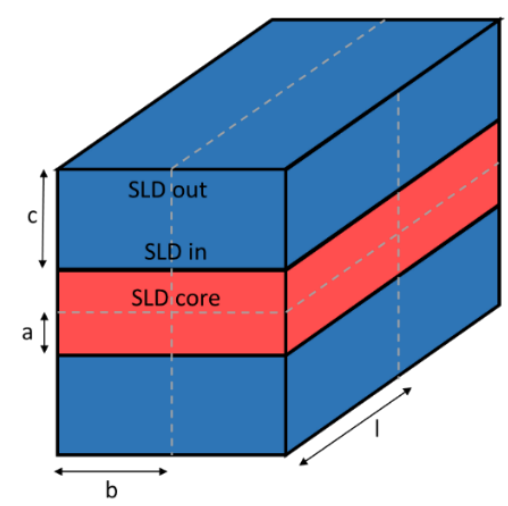

Figure S33. Schematic representation of Model 2 used in SAXS data fitting. Distances $a, b, c$ and $l$ represent half the core thickness, half the micelle width, the corona thickness and the micelle length respectively. SLD = scattering length density. 


\section{Supporting Information References}

1. Inam, M.; Cambridge, G.; Pitto-Barry, A.; Laker, Z. P. L.; Wilson, N. R.; Mathers, R. T.; Dove, A. P.; O'Reilly, R. K., 1D vs. 2D shape selectivity in the crystallization-driven self-assembly of polylactide block copolymers. Chem Sci 2017, 8 (6), 4223-4230.

2. Jones, E. R.; Semsarilar, M.; Blanazs, A.; Armes, S. P., Efficient Synthesis of Amine-Functional Diblock Copolymer Nanoparticles via RAFT Dispersion Polymerization of Benzyl Methacrylate in Alcoholic Media. Macromolecules 2012, 45 (12), 5091-5098.

3. Skey, J.; O'Reilly, R. K., Facile one pot synthesis of a range of reversible additionfragmentation chain transfer (RAFT) agents. Chem Commun 2008, (35), 4183-4185.

4. Boott, C. E.; Leitao, E. M.; Hayward, D. W.; Laine, R. F.; Mahou, P.; Guerin, G.; Winnik, M. A.; Richardson, R. M.; Kaminski, C. F.; Whittell, G. R.; Manners, I., Probing the Growth Kinetics for the Formation of Uniform 1D Block Copolymer Nanoparticles by Living Crystallization-Driven SelfAssembly. ACS Nano 2018, 12 (9), 8920-8933.

5. $\quad$ Finnegan, J. R.; Lunn, D. J.; Gould, O. E.; Hudson, Z. M.; Whittell, G. R.; Winnik, M. A.; Manners, I., Gradient crystallization-driven self-assembly: cylindrical micelles with "patchy" segmented coronas via the coassembly of linear and brush block copolymers. J Am Chem Soc 2014, 136 (39), 13835-13844.

6. Xu, J.; Zhou, H.; Yu, Q.; Manners, I.; Winnik, M. A., Competitive Self-Assembly Kinetics as a Route To Control the Morphology of Core-Crystalline Cylindrical Micelles. J Am Chem Soc 2018, 140 (7), 2619-2628.

7. Mencer, H. J.; Grubisic-gallot, Z., Influence of Solvent Polarity on Elution Volume in the Case of Polar Polymers. Journal of Liquid Chromatography 1979, 2 (5), 649-662. 